\title{
The use of different orientation cues in vernier acuity
}

\author{
MANFRED FAHLE \\ University Eye Clinic, Tübingen, Germany \\ and \\ JOHN P. HARRIS \\ University of Reading, Reading, England
}

\begin{abstract}
The role of orientation cues in vernier acuity was investigated in five experiments that varied the salience of different possible reference systems: (1) retinal, (2) subjective (vestibular), and (3) stimulusdefined ("intrinsic"). We compared the eight possible combinations of presence and absence of the three types of information about vertical by changing the orientation of the vernier stimulus, by changing the angle between the two segments of the stimulus, by reducing the stimulus to two dots rather than two lines, and/or by tilting the subject's head or by having the subject lie in a supine position. The results show that vestibular vertical plays no important role in vernier discriminations, whereas retinal vertical and, to a lesser degree, the intrinsic reference do. A control experiment excluded the possible role of a fourth possible reference system, the frame of the monitor. Our results are compatible with a model of vernier acuity detection based on oriented receptive fields with inhibitory surrounds. They show further that observers can produce, in the case of two-dot stimuli, an internal standard for comparison and detect deviations from this standard with high precision.
\end{abstract}

There are at least two kinds of cue in a vernier discrimination task, in which the subject has to judge the direction of the offset between two almost collinear lines. Subjects may make use of (integrated orthoaxial) position information, especially with abutting lines (Watt, Morgan, \& Ward, 1983). On the other hand, the task can be regarded as a judgment about the orientation of a virtual line through the stimulus (e.g., if dots are substituted for the line's endpoints; Andrews, Butcher, \& Buckley, 1973; Sullivan, Oatley, \& Sutherland, 1972). This orientation judgment may be made relative to some internal standard (e.g., "retinal vertical" or "vestibular vertical") or to the orientation of the lines forming the vernier stimulus themselves ("intrinsic vertical").

In most experiments on vernier acuity, possible effects of these kinds of reference orientation are confounded. That is, typically, the lines of the vernier stimulus are parallel and lie on one of the main cardinal orientations (horizontal or vertical), and so the orientation of the virtual line joining their ends could be compared with that of the lines themselves and/or to the subject's internal reference for that orientation. Previous attempts to investigate the po-

This work was funded by DFG Grants SFB 307 and TP A6 and by grants from the British Council and DAAD. This manuscript was prepared while M.F. was a Wiersma Visiting Professor at the California Institute of Technology, Pasadena. The authors thank H. Weller and A. Hildinger for participating as subjects. The authors are also grateful to $\mathrm{H}$. Weller for secretarial and graphical assistance and to three anonymous referees and the editor for improving the clarity of this paper. Correspondence should be addressed to M. Fahle, Department of Optometry and Visual Science, City University, 311-321 Goswell Rd., London, EC1V 7DD, England. tential contribution to vernier acuity of orientation information in the stimulus have kept the subject's head upright (for an exception, see Saarinen \& Levi, 1995) and the two vernier segments parallel to each other, but presented the vernier stimulus in different orientations. These experiments have been of two types. In one, the orientation of the vernier target varies from trial to trial (e.g., Harris \& Fahle, 1995; Watt \& Campbell, 1985). In these conditions, the implicit orientation of the line ends is reliably related from trial to trial to the orientation of the target segments (intrinsic), but not to the cardinal axes of space, and to subjective (vestibular) and retinal vertical. A typical finding is that thresholds obtained with stimulus orientation always vertical are lower than with orientation randomized, especially for short vernier targets. The second kind of study, in which target orientation is always the same within a condition, but varies between conditions, suggests that thresholds are higher for obliquely oriented targets than for vertical targets (Corwin, Moskowitz-Cook, \& Green 1977), even after extended practice (Morgan, 1991). In principle, the rise in thresholds with randomized orientations or oblique presentation could result either because the vernier target is not close in orientation to a subjective (vestibular) vertical or horizontal or because the vernier target is obliquely oriented on the retina (or, in the case of variable orientation, because targets in a constant orientation can be processed more reliably than those whose orientation is variable).

The present study examined the effects of stimulus orientation on the retina, of changes in the subject's posture, and of another possible source of orientation information in vernier tasks - namely, that provided by the vernier seg- 


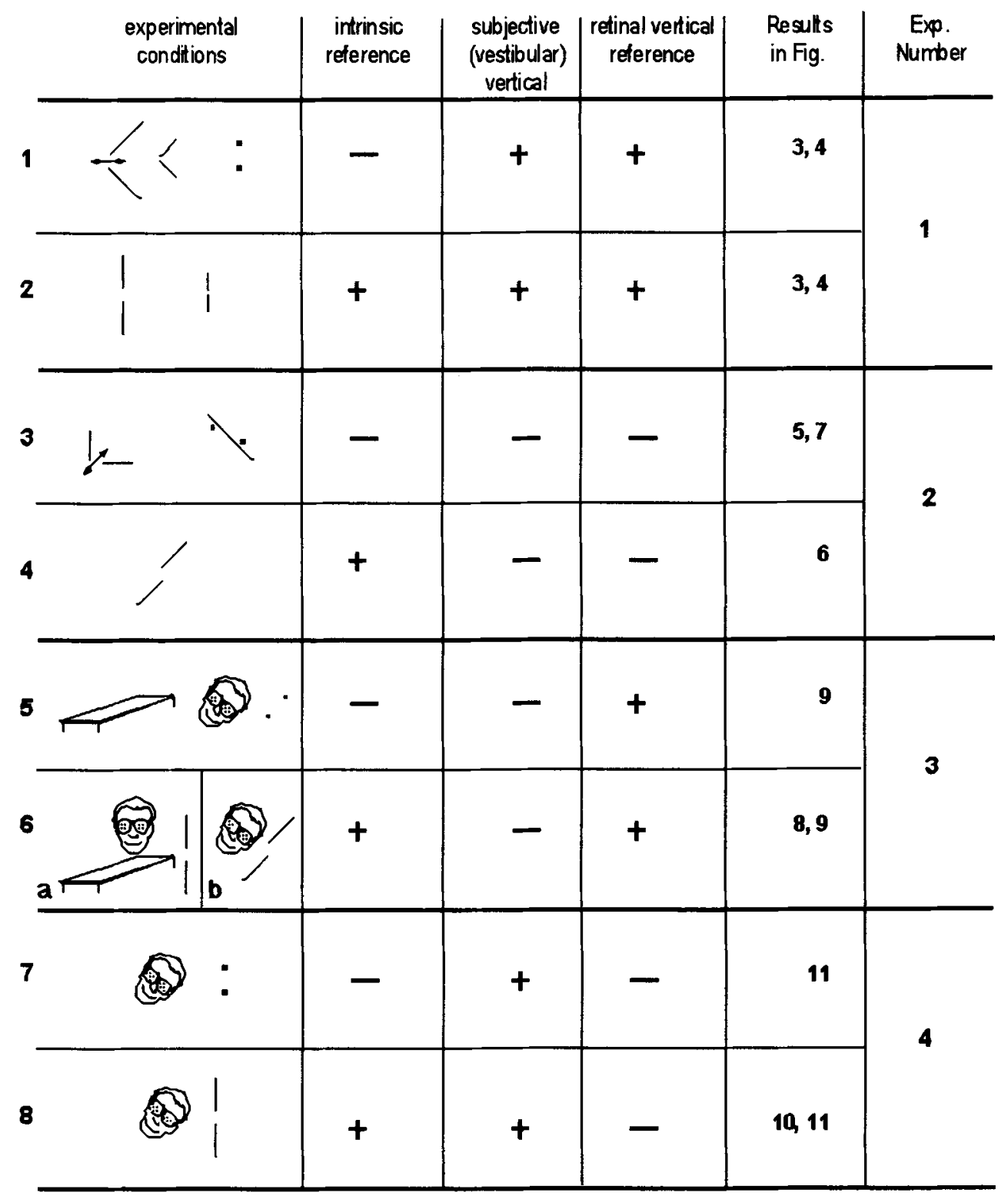

Figure 1. Guide to the experimental conditions. Each of Experiments 1-4 contained two conditions that differed regarding the presence $(+)$ or absence $(-)$ of the strong intrinsic frame of reference. In each condition, the stimulus either did $(+)$ or did not $(-)$ coincide with vestibular and/or retinal vertical. The conditions of each experiment are symbolized in the left-hand column; the second column from the right indicates the figure(s) that show(s) the results. All possible permutations of the presence $(+)$ and absence $(-)$ of all three components were investigated. In Condition $6 \mathrm{a}$, the subject was lying in a supine position on a hospital trolley.

ments themselves. Figure 1 provides a schematic guide to the stimuli used, the type of reference present in the stimulus, and the figures in which the results are to be found. In Experiment 1, with the subject's head upright, the individual segments of a target were either vertical or rotated in opposite directions by various amounts to form a chevron, so that the rotated versions did not provide an explicit intrinsic reference orientation (Figure 1, Conditions 1 and 2). The task was always to judge the direction of the horizontal offset between the near ends of the line segments. In a control condition, we used dots rather than line elements. Observers had strong gravitational (vestibular) and retinal references against which to discriminate the direction of offset while the intrinsic reference was either present (line verniers) or absent (chevrons, dots).

We repeated the first condition in the first part of Experiment 2, but with the whole stimulus rotated by $45^{\circ}$ (Figure 1 , Condition 3). In the second part of Experiment 2 , we repeated the second condition for oblique stimulus orientations. The subject's head was upright, and conventional vernier targets 
(with parallel segments) were presented at the same orientations as those used for the individual segments in the first condition (Figure 1, Condition 4), since it was felt important to measure the size of any oblique effect in our conditions with our observers. We hoped that the comparison of thresholds from the two kinds of experiment would show whether the effects of changing segment orientation could be ascribed to some aspect of the oblique effect on vernier acuity.

In Experiment 2, neither gravitational nor retinal vertical provided strong references for discriminating between the two directions of offset since stimulus orientation was clearly different from vertical in most presentations and since the offset was in an oblique direction. Again, an intrinsic reference was either present (line verniers) or absent (chevrons, dots).

In Experiment 3, the effects of varying the orientation of the subject's head as well as the orientation of the stimulus were examined. The aim of this part of the study was to dissociate the effects of retinal vertical from those of vestibular vertical. In one set of conditions, the vernier stimuli, consisting either of lines or of dots, were oblique on the monitor, and the observer's head was rotated by $45^{\circ}$. In another, the subject lay supine and viewed the monitor, on which the line or dot verniers were vertical, through a mirror (Figure 1, Conditions 5 and 6). In these conditions, the orientation of the vernier no longer coincided with the normally available cue of gravitational vertical, but it still coincided with retinal vertical.

In Experiment 4, a two-dot vernier stimulus or a vernier with parallel line elements was always vertical on the monitor, with the subject's head tilted $45^{\circ}$ from vertical (Figure 1, Conditions 7 and 8). In these conditions, vernier orientation coincided with vestibular vertical, but not with retinal vertical.

The intention of these manipulations was to vary the relevant vestibular information available to the subject while making vernier judgments, in addition to varying the retinal orientation of the stimuli. Similar manipulations in most other studies have shown that meridional variations in contrast sensitivity for gratings (Banks \& Stolarz, 1975; Lennie, 1974), vernier acuity (Corwin et al., 1977; Saarinen \& Levi, 1995), and orientation discrimination (Orban, Vandenbussche, \& Vogels, 1984) occur in retinal and not in gravitational coordinates. There are, however, exceptions to this rule (Buchanan-Smith \& Heeley, 1993). In the control experiment (Experiment 5), the possible effects of cyclotorsion of the eyes and of the frame of reference supplied by the edges of the monitor were investigated.

\section{EXPERIMENT 1 \\ Strong Gravitational and Retinal Reference With Varied Intrinsic Orientation Information}

In Experiment 1, in which the subject's head was always upright, the orientation of the lines forming a vernier stimulus (though constant within a condition) varied systematically between conditions, while the direction of the offset between the line ends remained constant. In these conditions, the "parent" stimulus was two vertical lines separated by a vertical gap. Three aspects of this stimulus varied to produce the family of stimuli used in the experiment. The size of the vertical gap between the line ends varied, as did the orientation of the lines by rotation in opposite directions around their endpoints at each end of the gap. This means that the form of the stimuli was that of a chevron with a gap at its center (Figures $2 a$ and $2 b$ ). Thus, the reference information usually supplied by the orientation of the lines themselves varied, and, although the vertical orientation was still implicitly given, there was no explicit intrinsic vertical orientation presented. The direction of the offset between their nearer line ends was always horizontal. On Wilson's (1986) line element model of vernier acuity, this manipulation should impair acuity, since the different orientations of the vernier segments would make them less optimal stimuli for the oriented filters with elongated excitatory centers and flanking inhibitory surrounds on which the model depends (see Figures $2 \mathrm{c}$ and $2 \mathrm{~d}$ ). To check whether tilting the lines of the individual vernier segments impairs discrimination of the direction of the offset between the line ends (e.g., by giving misleading orientation information rather than by giving no orientation information at all), conditions were included in which only the line ends (i.e., small dots) were presented. These dot stimuli contained no oriented line elements and so did not contain "misleading" orientation information, but they lacked intrinsic orientation information altogether. The third stimulus variable was line length. The aim of this variation was to check whether the potentially misleading orientation information given by tilted lines would be more salient if the lines were longer.

\section{Method}

Apparatus. Stimuli were presented on the screen of a display oscilloscope (Tektronix 608), with green (P31) phosphor, by a 32-bit microcomputer via fast 16 -bit D/A converters. Stimulus luminance, as measured on a homogeneous test field of a point density identical to that of the stimuli, was around $200 \mathrm{~cd} / \mathrm{m}^{2}$ (corresponding to a linear luminance around $0.07 \mathrm{~cd} / \mathrm{m}^{2}$; Westheimer, 1985), and the luminance of the remainder of the screen was around $0.1 \mathrm{~cd} / \mathrm{m}^{2}$. Viewing was binocular, at a viewing distance of $2.5 \mathrm{~m}$, giving a vernier target line width around $1.5^{\prime}$ of arc. The room was so dimly lit that, apart from the monitor, no details of the room could be discriminated. The subject's head was stabilized in the upright position by a chinrest and brow bar in most of the experiments.

Procedure. Data were collected using an adaptive method of parameter estimation by sequential testing (PEST; Taylor \& Creelman, 1967), which terminated the test run when the step size fell to some criterion (rarely) or when 50 stimuli had been presented. Subsequently, thresholds (the difference between the $50 \%$ and $75 \%$ points of the psychometric function) were calculated by means of probit analysis (Finney, 1962). The results of both methods usually were satisfactorily similar. Data points in the graphs represent the means of 3-6 of these runs. Statistics were performed on a Power Macintosh using Statview and SuperANOVA software.

Stimuli. Examples of the stimuli used are shown in Figure 2. Basically, the stimuli consisted of two lines of different length that were displayed at different angles symmetrically around the horizontal meridian. The angles indicated on the abscissae of the graphs indicate the angle between the vernier elements and the parent straight stimulus. Thus, at $45^{\circ}$, both elements deviated by $45^{\circ}$ from the ver- 

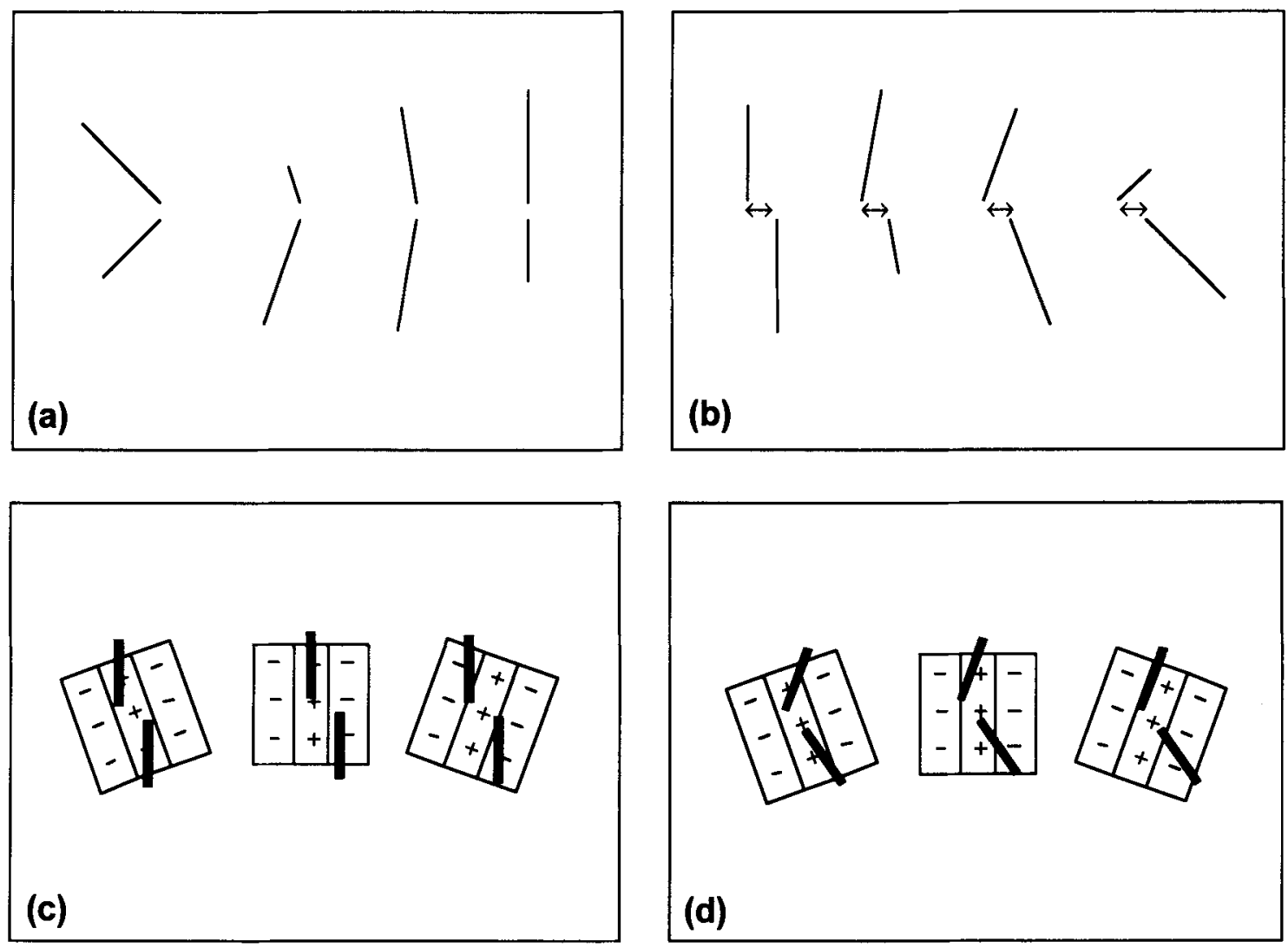

Figure 2. Examples of the stimuli used in Experiment 1. Angles indicate the angle between each element of the "chevronized" stimulus and the "parent" straight stimulus. Negative angles on the $x$-axes in Figures 3-15 refer to tilting to the left, as in Panel a where the stimuli had no offset. Positive angles refer to tilting to the right, as in Panel b, where stimuli were offset in addition to being tilted or straight. The offset in these stimuli was always in the horizontal direction. Panel c: Vernier stimuli were superimposed on receptive fields with excitatory centers and inhibitory surrounds. A vernier offset (of the lower line segment) to the right maximally excited a receptive field tilted to the left, since it did not activate the inhibitory surround. Panel d: A chevronized vernier could not maximally activate any of the oriented receptive fields.

tical, forming a right angle. Stimulus orientation was constant within each PEST run. The inner endpoints of the adjacent ends of the lines were offset in the horizontal direction by a distance that was varied by the PEST procedure. Mean line length was either $9^{\prime}$ or $28^{\prime}$ of arc, varying randomly, with equal probability, between $2^{\prime}$ and $17^{\prime}$ of arc $\left(\right.$ mean $=9^{\prime}$ ) and between $6^{\prime}$ and $50^{\prime}$ of $\operatorname{arc}\left(\right.$ mean $\left.=28^{\prime}\right)$, respectively, to ensure that the observers could base their judgment about the direction of offset on only the horizontal displacement between the adjacent endpoints, rather than on the farther ends of the lines or on the center of the respective luminance distributions. Control experiments were performed using two dots (whose diameter equaled the line width) positioned at the nearer line ends rather than lines, to see whether tilting the lines decreased performance for the vernier task relative to dot stimuli. The size of the vertical separation between the adjacent line ends (gap size) was either $5^{\prime}, 10^{\prime}$, or $15^{\prime}$ of arc for all experiments. For $100 \mathrm{msec}$ immediately prior to the presentation of a stimulus, the corner of a rectangular frame appeared at the corners of the display. This served to guide accommodation and fixation and acted as a warning signal. Presentation time in this and subsequent experiments was 2 sec.

Subjects. Four subjects ( 2 males, 2 females; age range $=44-52$ years) volunteered to participate in the experiments. All were experienced psychophysical observers and had normal or corrected-tonormal vision. Two of the subjects (M.F. and J.H.) were the authors; the other 2 subjects (H.W. and A.H.) were naive to the hypotheses under test.

\section{Results}

Vernier detection thresholds for the individual observers, as well as their means and standard errors, are shown in Figure 3 for the lines with a maximal length of $50^{\prime}$ of arc and in Figure 4 for the lines with maximal length of $17^{\prime}$ of arc. Thresholds were clearly lowest for an angle of $0^{\circ}$ (i.e., for vertical orientation of both elements of the vernier): From the length (2) $\times$ gap size (3) $\times$ angle (7) $\times$ subject (4) analysis of variance (ANOVA), the effects of changing angle were highly significant $[F(6,18)=6.22$, $p<.001]$. Thresholds in this condition were well within the hyperacuity range, and they corresponded approximately to standard vernier thresholds with elements of equal size (in the present experiment, the lines were of unequal randomly varied lengths). With increasing deviation of the vernier elements from the vertical, and, hence, from straightness (called increasing angle in this paper), thresholds increased moderately for all observers by an average 

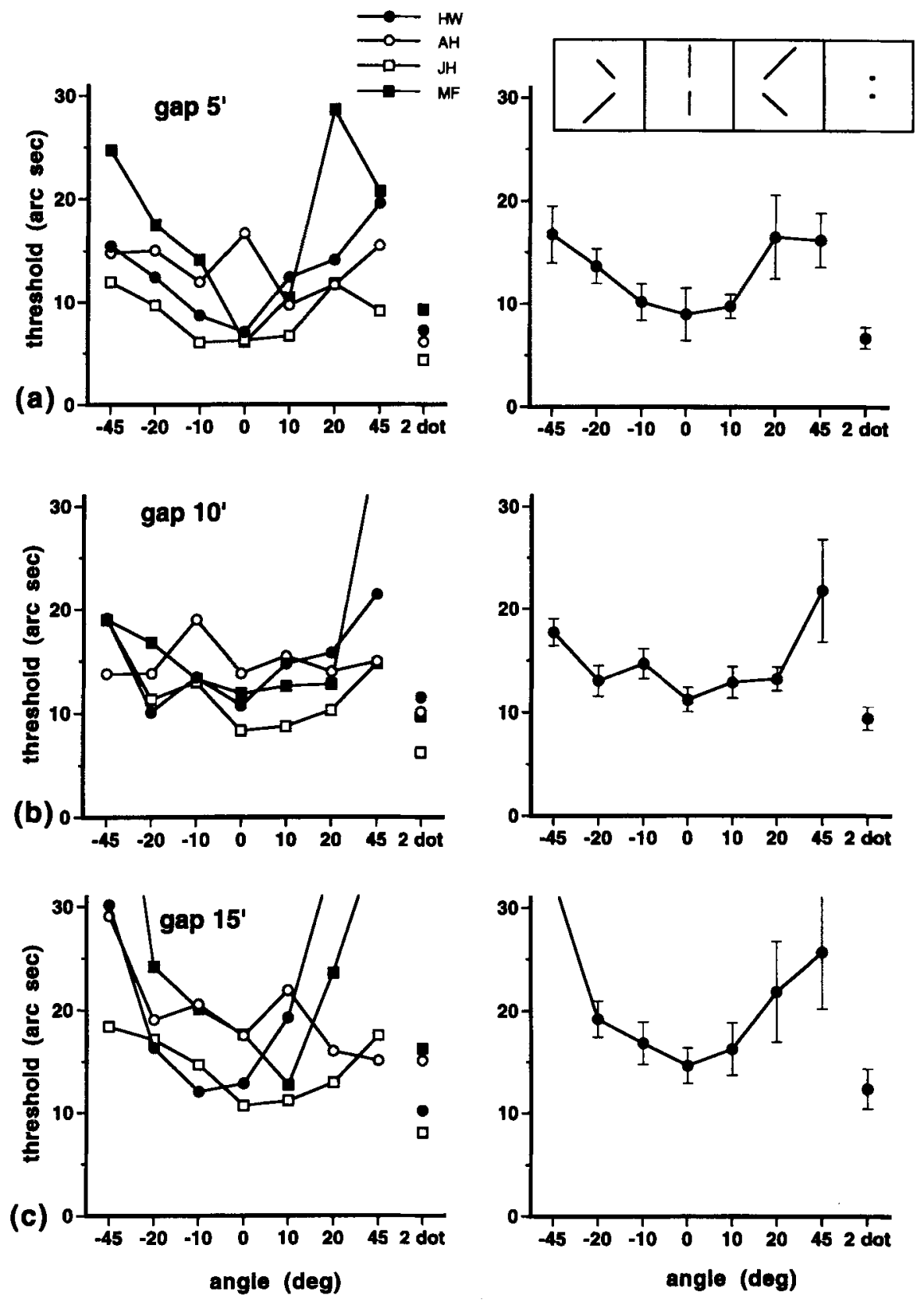

Figure 3. Vernier detection thresholds for lines with a maximal length of $50^{\prime}$ of arc as a function of the angle between vertical and each of the two segments of the stimulus. The left-hand panels show individual results of the 4 subjects. Control measurements (isolated data points on the right) were taken using dots rather than lines. The right-hand panels show the means and standard errors of the means (vertical bars) of all 4 subjects. Isolated data points on the right indicate results for stimuli consisting of two single dots.

factor of 2 between vertical and oblique $\left(45^{\circ}\right)$ orientation. Results, as expected, were very similar for positive angles (upper line tilted to the right) and negative angles (upper line tilted to the left; see Figure 3 ). The effect of increasing gap size for both the $2^{\prime}-17^{\prime}$ and the $6^{\prime}-50^{\prime}$ stimuli, was to raise thresholds, both when the vernier elements were vertical and when they were oblique $[F(2,6)=19.16$, $p<.003]$. There was no significant interaction between gap size and angle $[F(12,36)=1.35, p<.25]$. This general finding of higher thresholds with increasing gap size above 5 arc min is well in line with previous work (Watt, 1984; Westheimer \& McKee, 1977).

In the control condition with two points (falling at the positions of the adjacent line ends) rather than two line elements, thresholds turned out to be similar to those with vertical vernier elements (see the isolated data points on 

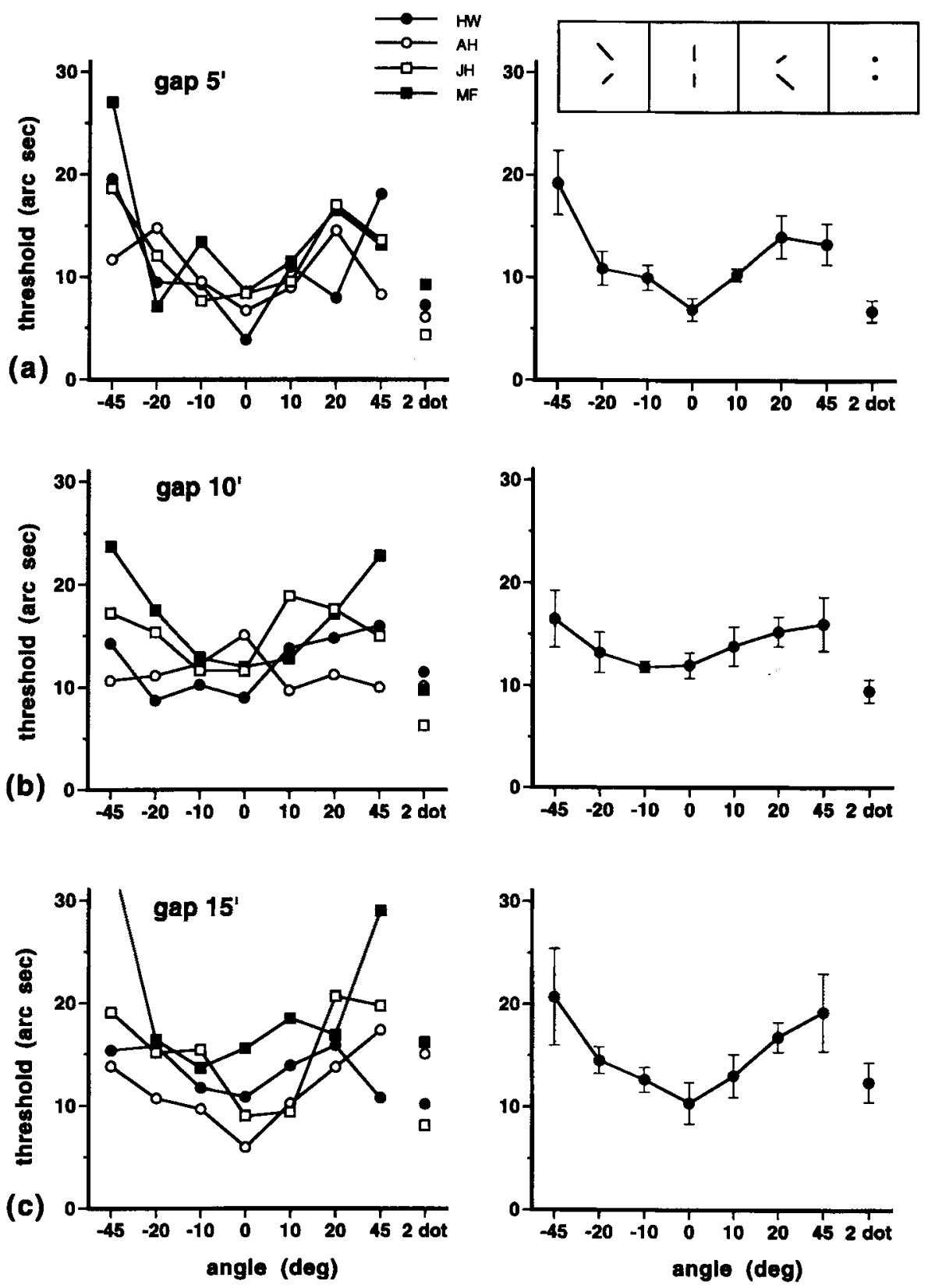

Figure 4. Vernier detection thresholds for lines with a maximal length between $2^{\prime}$ and $17^{\prime}$ of arc as a function of the angle between vertical and each of the two elements of the stimulus. The lefthand panels show individual results of the 4 subjects. The right-hand panels show the means and standard errors of the means (vertical bars) of all 4 subjects. Isolated data points on the right indicate results for the two-dot stimuli.

the right-hand side of each panel of Figures 3 and 4). As for the thresholds with line elements, these thresholds for two dots rose with the size of the interdot gap.

The effects of varying line length were not significant $[F(1,3)=2.22, p<.25]$, though there was a significant interaction between line length and gap size $[F(2,6)=14.03$, $p<.005]$. Inspection of Figures 3 and 4 suggests that this interaction reflects performance on the largest gap size, on which performance was worse for the longer lines.

\section{Discussion}

It is not clear why performance for longer lines was worse than for shorter lines at large gap sizes. This difference might have been expected at the larger angles, if the misleading orientation information provided by the longer lines was more salient. However, it was also present for the vertical verniers, suggesting some other origin for the effect.

Tilting the vernier line elements away from vertical into a chevron-like format clearly impaired vernier acuity. The 
low thresholds for the two-dot conditions suggest that tilting the vernier line elements in opposite directions did not simply remove a source of information but added misleading or inhibitory information. This finding is in good agreement with a model that incorporates oriented bar detectors as the neural basis of vernier offset detection (Wilson, 1986; see Figure 2). As long as the stimulus is projected completely on the excitatory center of the receptive field, activation of the detector is maximal. Offset verniers maximally activate receptive fields whose axis does not correspond to the long axis of the vernier elements (Figure 2c). The vernier stimulus with rotated elements is unable to maximally activate one of the detectors since the inhibitory surrounds of straight bar detectors--usually providing information about vernier offset-are always activated, decreasing the activation of the detectors (Figure $2 \mathrm{~d}$ ).

Moreover, the results indicate that the stimulus-defined intrinsic vertical does not play an important role in vernier acuity when the retinal vertical reference is present, since two dots yield thresholds comparable to vertically oriented vernier lines (see Westheimer \& McKee, 1977).

It might be thought that the increases in thresholds found in this experiment are somehow connected to the randomization of segment length, even though this was apparently not a relevant cue in performing the task. Randomizing relevant cues (such as the orientation of collinear vernier targets) certainly does increase thresholds (Watt \& Campbell, 1985; the present Experiment 5). However, if length randomization were important in this experiment, one might expect higher thresholds than usual for the collinear stimuli with segments of randomly chosen length. The low thresholds found here for such targets (see Figure 4a), comparable to thresholds in the literature for vertical verniers with segments of equal length, suggest that such effects are small if they occur at all. Similarly, if changes of segment orientation are irrelevant, the graphs in Figures 3 and 4 should be horizontal straight lines, and they are clearly not. Thus, we argue that any effects of randomizing the irrelevant cue segment length in this experiment were small.

As outlined in the introduction, orientation information is supposed to be a strong cue, especially for short vernier targets: Thresholds for these stimuli increase most strongly with variable orientation of the vernier, relative to fixed orientation. We do not, however, find a significant difference between the orientation dependence of long stimuli (Figure 3) versus short stimuli (Figure 4). Hence, the increase of thresholds with rotation of the vernier elements seems to be caused by neuronal mechanisms different from the ones found in the study by Watt and Campbell (1985), in which orientation differed between individual stimulus presentations. Similarly, implicit orientation information is considered to be a relatively stronger cue in stimuli with a smaller gap between the two segments of the vernier than for stimuli with a larger gap. Again, our data do not show a significant difference between the orientation dependence of stimuli with small versus large gaps, though stimuli without any gap might yield a different pat- tern of results. Hence, the loss of implicit orientation information due to the "chevronization" seems not to be the main reason for the increase of thresholds with increasing deviation of the vernier segments from straightness. An alternative explanation for this finding is that the decrease of performance with oblique orientation is not an effect primarily caused by removal of orientation cues but that the effect is caused by less precise neuronal analysis of stimuli not displayed along the main cardinal axes. This possibility was addressed in Experiment 2.

\section{EXPERIMENT 2 \\ Weak Gravitational and Retinal Reference With Varied Intrinsic Orientation Information}

Experiment 1 showed that performance declined as the angle between the vernier segments deviated from $0^{\circ}$ (i.e., from parallel orientation). Because performance with two vertically separated dots was comparable to that when the vernier segments were vertical, the intrinsic reference orientation given by the lines in that condition cannot be adding much useful information. It could be that performance falls as the vernier segments are tilted because the oblique lines provide a misleading reference and so actively impair acuity. However, another possibility is that, as the lines deviate more from vertical (and so become more oblique), the precision with which their orientation is perceived decreases, as does the precision with which their offset can be detected, if we assume that the localization of the line elements is made perpendicularly to their orientation. To discriminate between these two possible explanations and to assess the influence of intrinsic orientation information in the (near) absence of close gravitational and retinal orientation information, we performed two tests. The first one repeated Experiment 1 but with the stimulus rotated by $45^{\circ}$. Hence, at $0^{\circ}$ deviation, this stimulus corresponds to a straight vernier, oriented at $45^{\circ}$ from the vertical; at $45^{\circ}$ deviation, one of the elements of the vernier is oriented vertically, while the other element is oriented horizontally. For the second test, we varied the orientation of a collinear vernier target and of the two-point vernier (i.e., we tested the "oblique effect").

It is already known that vernier acuity becomes worse when a collinear stimulus is oriented obliquely (Corwin et al., 1977). However, we needed to know the size of this effect in our conditions (i.e., with variable length of the vernier elements and for our observers) in order to judge whether it could account quantitatively for the results of Experiment 1. Since the differences between the $50^{\prime}$ and the $17^{\prime}$ verniers were apparent only at the largest gap in Experiment 1, measurements in Experiment 2 were made only with the longer verniers.

In view of the good performance in discriminating the direction of a horizontal offset between two dots in Experiment 1 , the effect of reducing the vernier lines to their near endpoints (two dots) was also examined. In effect, this changes the direction of the offset between the dots from horizontal to one of a range of oblique orientations, as was 
the case with the oblique vernier lines, and leaves no retinal, gravitational, or intrinsic orientation information.

\section{Method}

The apparatus, procedure and the 4 observers were the same as those in Experiment 1 . The monitor was rotated by $45^{\circ}$ relative to that in Experiment 1.

The stimuli were similar in most respects to those used in Experiment 1 . The line elements varied in length randomly between $6^{\prime}$ and $50^{\prime}$ of arc. The gap between the nearer ends of the lines was $5^{\prime}, 10^{\prime}$, or $15^{\prime}$ of arc. In contrast to Experiment 1 , the basic stimulus in the first part of Experiment 2 was a collinear line vernier tilted at $45^{\circ}$ from vertical. From this parent stimulus, a family of stimuli was produced similar to the ones in Experiment 1 (i.e., chevron-like stimuli). Stimuli in the second part of Experiment 2 were always collinear, and the orientation of the whole vernier varied systematically. We presented stimuli in seven orientations: vertical, three orientations clockwise from vertical $\left(10^{\circ}, 20^{\circ}\right.$, and $\left.45^{\circ}\right)$, and three orientations counterclockwise from vertical $\left(-10^{\circ},-20^{\circ}\right.$, and $\left.-45^{\circ}\right)$. To measure the oblique effect with dots, the lines were simply reduced to their nearer endpoints by setting their length to be equal to their width, but the same presentation orientations were used as with line verniers.

\section{Results}

The replication of Experiment 1, but with the stimuli rotated by $45^{\circ}$ counterclockwise (i.e., not coinciding with vestibular or retinal vertical, and without a stimulus-defined vertical), yielded results whose pattern was similar to that of Experiment 1 (Figure 5). Lowest thresholds were obtained for straight, collinear stimuli, and thresholds increased with increasing deviation of the two elements of the stimulus from straightness somewhat more strongly than in Experiment 1. In all conditions, thresholds were roughly a factor of 2 worse than those found in Experiment 1.

The results for the collinear line stimuli are shown in Figures $6 \mathrm{a}, 6 \mathrm{~b}$, and $6 \mathrm{c}$. Mean thresholds for the vertical stimulus were between 7 " and 11 " of arc for all gap sizes, and they increased with gap size. In general, this pattern of performance was maintained as the vernier target became more oblique. Roughly, thresholds rose by a factor of about 2 as orientation changed from vertical to $45^{\circ}$ oblique. A repeated measures ANOVA with factors of orientation (7) $\times$ gap size $(3) \times$ subject $(4)$ yielded highly significant effects of stimulus orientation $[F(6,18)=10.95, p=.0001]$ and gap size $[F(2,6)=34.8, p=.0001]$.

Data for discriminating the direction of the offset between the dots are shown in Figures $7 \mathrm{a}, 7 \mathrm{~b}$, and $7 \mathrm{c}$. Note that, here, additional measurements were taken for an angle of $90^{\circ}$, corresponding to a horizontal orientation of the dot stimuli. These graphs are similar to those for the lines (Figures $6 a, 6 b$, and $6 \mathrm{c}$ ), but the data are more variable. The lowest thresholds were found when the offset was horizontal, and they increased with angle up to $45^{\circ}$ (i.e., oblique orientation) to improve again for $90^{\circ}$ (i.e., horizontal orientation of the dot stimuli). From the orientation (7) $\times$ gap size (3) $\times$ subject (4) repeated measures ANOVA, the effects of changing orientation were significant $[F(6,18)=4.70, p=.0004]$. Thresholds increased significantly with gap size $[F(2,6)=12.6, p=.0001]$; however, collapsed across orientations, thresholds for the dot stimuli were not significantly different from those for the line stimuli $[F(1,3)=4.66, p=.12]$. In addition, the apparently steeper increase in thresholds with deviation from vertical for the dot stimuli, relative to the line stimuli, was not significant [for the stimulus type $\times$ orientation interaction, $F(12,36)=1.63, p=.2]$.

The results from the dot and line stimuli were compared in a stimulus type (2) $\times$ orientation (7) $\times$ gap size (3) $\times$ subject (4) repeated measures ANOVA. The interactions between stimulus type and gap size, between stimulus type and orientation (see above), between gap size and orientation, and between stimulus type, gap size, and orientation all failed to reach significance $(.09<p<.58)$.

\section{Discussion}

As can be seen in Figures 3, 4, and 5, collinear verniers, indicated by $0^{\circ}$, always yielded best results, with thresholds increasing with angle. This pattern of results indicates that the increase in thresholds with increasing deviation of the vernier stimulus from straightness was not caused primarily by the deviation of the elements' orientation from vertical. On the contrary, Figure 5 demonstrates that thresholds increased as the orientation of the elements changed from oblique to vertical and horizontal. This is in line with the finding that lowest performance (i.e., highest thresholds in Experiment 1) was obtained with vernier elements oriented at $45^{\circ}$, a secondary cardinal axis (see Regan $\&$ Price, 1986). Hence, the main reason for the increase of thresholds in chevron-like stimuli, as found in Experiment 1 , is the deviation of these stimuli from straightness. As with the stimuli in Experiment 1, gap size had a relatively weak effect.

A surprising feature of the results is the fact that the increase of thresholds with angles deviating from $0^{\circ}$ is roughly comparable in Figures 6 and 7. Since the data in Figure 6 were measured using conventional verniers, the orientation of the offset was explicitly represented within each stimulus. In the case of the dots from which the data in Figure 7 were obtained, however, the direction of the offset could not, in principle, be recovered from a single stimulus presentation. This could only be done by some kind of comparison across a number of stimulus presentations, and, hence, it involves some form of visual memory. Thresholds based on this comparison of stimuli with an internal standard are almost (but not quite) as low as those that do not involve such a standard. The adaptive method of threshold estimation was helpful here since differences between stimuli were high initially, allowing the subjects to readily synthesize a zero point.

\section{EXPERIMENT 3 Strong Retinal Reference With Weak Gravitational and Varied Intrinsic Orientation Information}

Varying the orientation of the separate line elements of a vernier target could in principle change two local sources of information used in the vernier task. As suggested above, one is the reference orientation provided by 

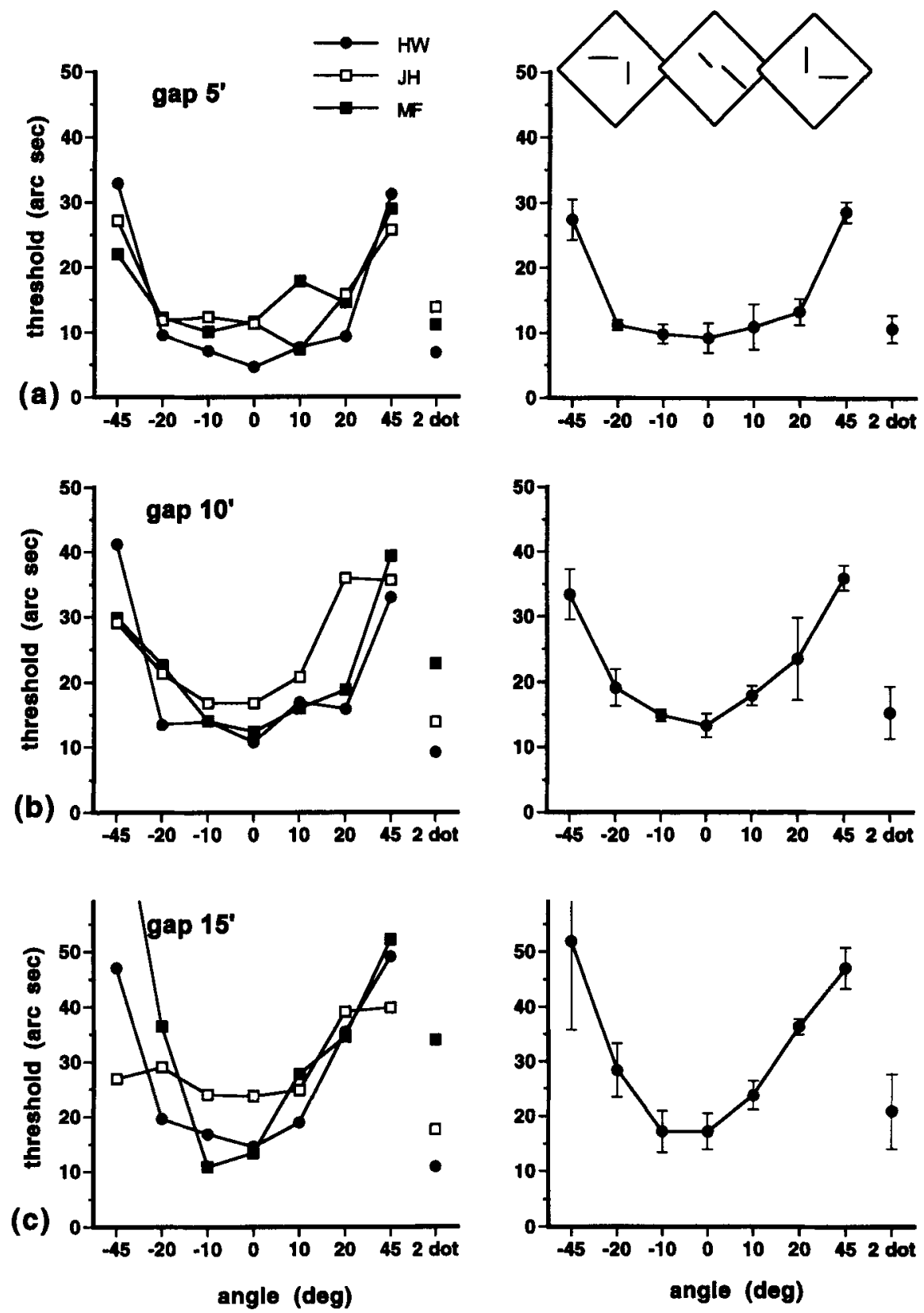

Figure 5. Thresholds for lines with a maximal length between $2^{\prime}$ and $17^{\prime}$ of arc as a function of angle between $45^{\circ}$ counterclockwise and each of the two segments of the stimulus (i.e., replication of the experiment whose results are presented in Figure 4 with the monitor rotated by $45^{\circ}$ counterclockwise). The left-hand panels show the individual results. The right-hand panels show the means and standard errors of the means. Isolated data points on the right indicate results for the two-dot stimuli.

the line elements themselves, and the other is the precision with which the lines and their orientation are localized. When the whole vernier changes orientation, only the second variation occurs. However, tilting the whole vernier changes its relation to a more global frame of referencenamely, perceived or subjective vertical. When the head is upright, two sources of information that could contribute to this reference frame are retinal vertical and vestibular vertical. To examine the relative contributions of these factors to vernier acuity, we varied both the posture of the subject (in Experiment 3 ) and the orientation on the retina (in Experiment 4) of both a conventional line and a twodot vernier target.

There were three posture conditions: sitting upright, sitting tilted (so that the head was tilted sideways by $45^{\circ}$ ), and lying supine. The vernier stimulus could be either vertical 

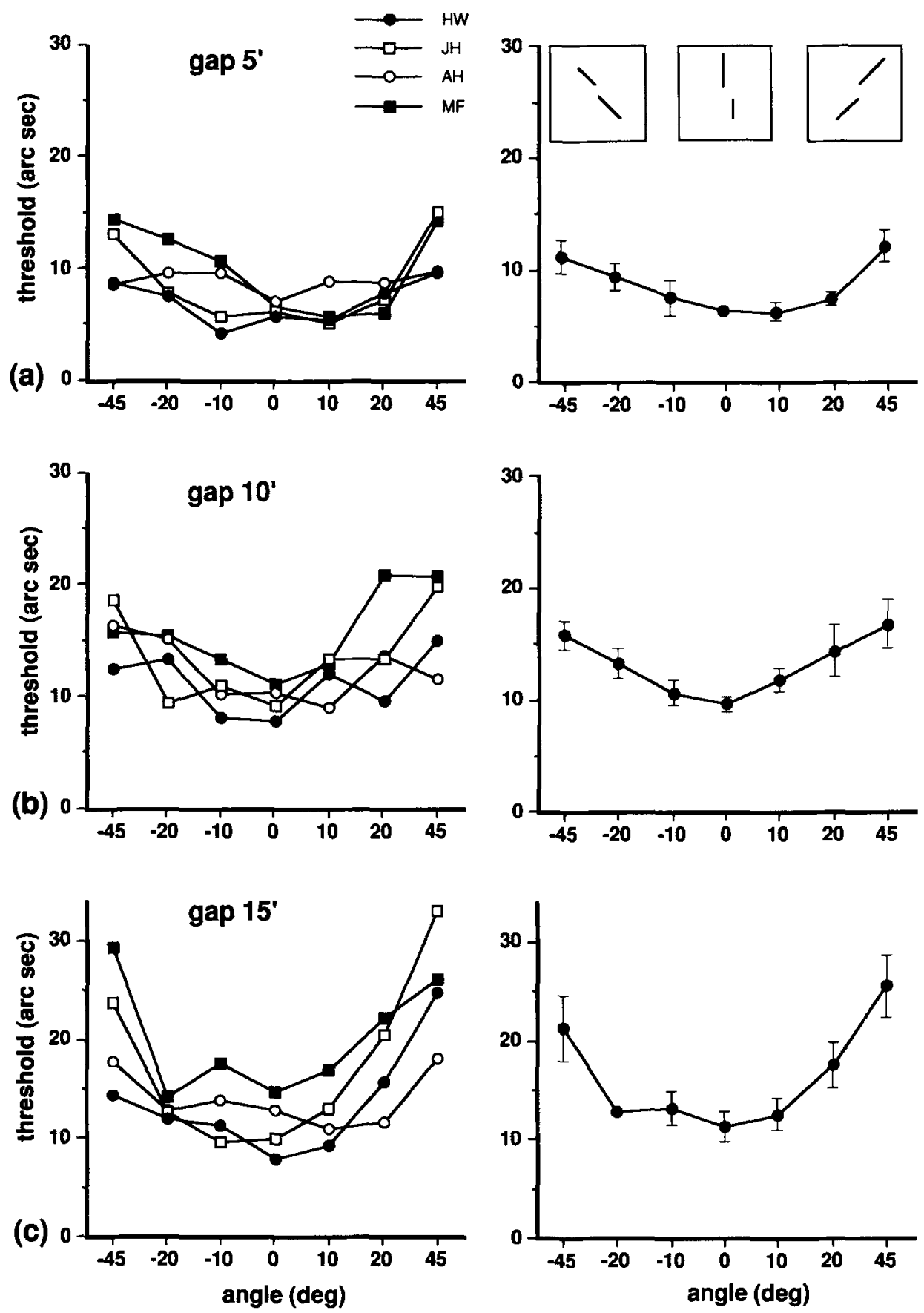

Figure 6. Vernier thresholds for line vernier targets as a function of orientation. Orientation varied between vertical $\left(=0^{\circ}\right)$ and oblique $\left(=45^{\circ}\right.$ tilted to the right, or $-45^{\circ}$ tilted to the left). The left-hand panels show results of individual subjects. The right-hand panels show the means and standard errors of the means of all 4 subjects.

on the monitor screen or tilted $45^{\circ}$ from vertical. Within each posture condition, therefore, the stimulus could be either vertical on the retina (in Experiment 3 ) or tilted at $45^{\circ}$ to retinal vertical (in Experiment 4), a meridian far less perceptually salient than the horizontal and vertical ones (Regan \& Price, 1986). In Experiment 3, all stimuli were always oriented vertically on the retina; hence, there was a strong retinal reference but only a weak gravitational reference.

\section{Method}

Apparatus. The display and the psychophysical procedure were the same as those in Experiments 1 and 2. The subjects' posture was 

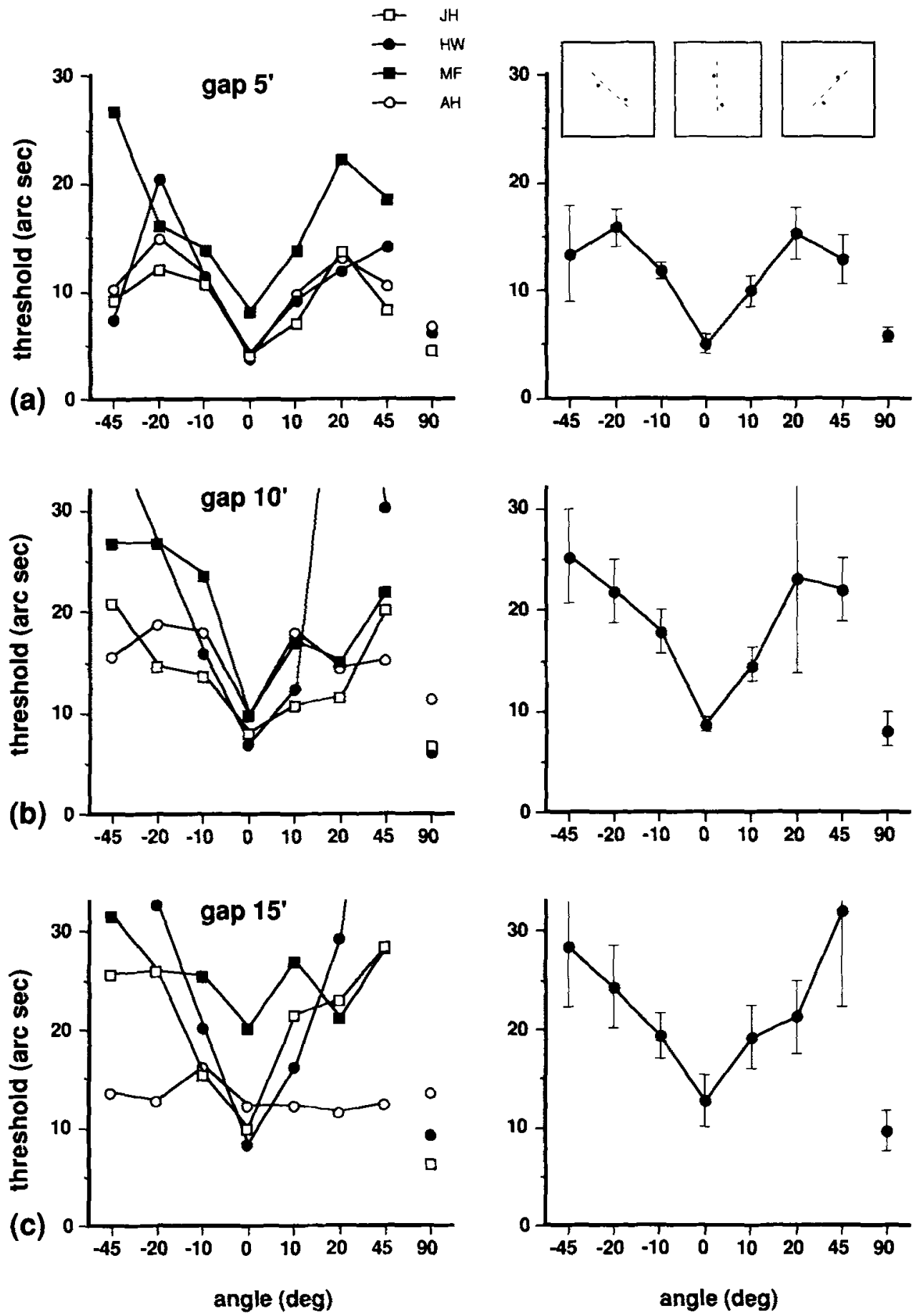

Figure 7. Vernier thresholds for two-dot targets as a function of orientation. Orientation varied between vertical $\left(=0^{\circ}\right)$ and oblique $\left(=45^{\circ}\right.$ tilted to the right, or $-45^{\circ}$ tilted to the left). The left-hand panels show results of individual subjects. The right-hand panels show the means and standard errors of the means of all 4 subjects.

altered as follows. In the head-upright condition, they sat in the same chair as was used in Experiments 1 and 2 . In the head-tilted condition, they sat leaning sideways on a chair with a low back to which a headrest had been fixed, and they aligned the head with a $45^{\circ}$ line on a protractor. In the supine condition, the subjects lay on their backs on a bed with the imaginary line joining the centers of their pupils at $90^{\circ}$ to the midline of the trunk, and they viewed the display through a mirror above the head. The subjects usually had about $2-3 \mathrm{~min}$ to adjust to the new head positions.

Stimuli. The vernier targets were always collinear. In different conditions, they had lengths between $2^{\prime}$ and $17^{\prime}$ of arc $\left(\right.$ mean $\left.=9^{\prime}\right)$ or between $6^{\prime}$ and $50^{\prime}$ of arc $\left(\right.$ mean $\left.=28^{\prime}\right)$. Line length was varied here 

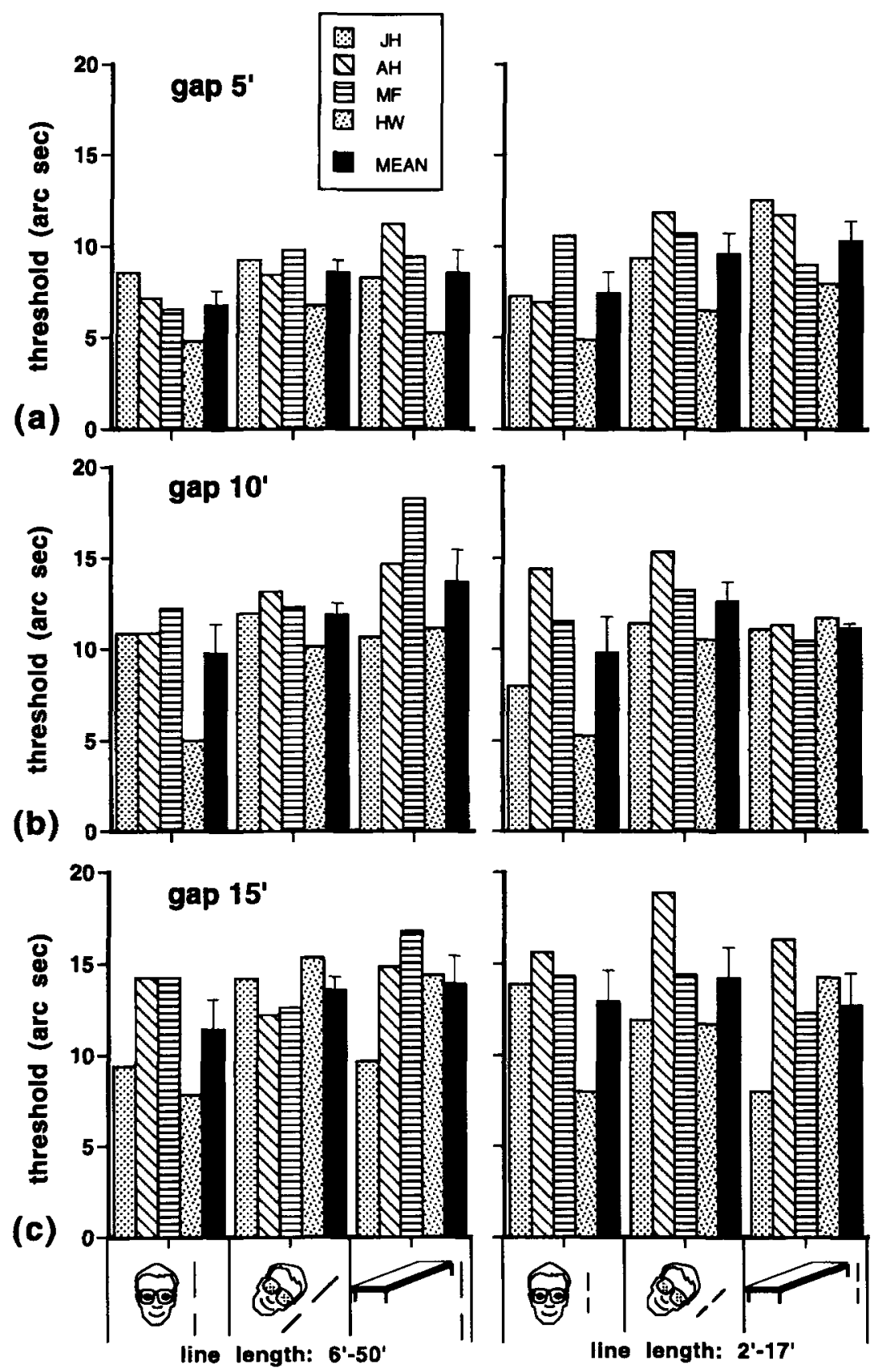

Figure 8. Vernier thresholds for different postures of the subjects and different orientations of the stimuli. Stimuli were always vertical on the retina. Line length varied between $6^{\prime}$ and $50^{\prime}$ (mean $=28^{\prime}$ ) of are for the three leftmost conditions and between $2^{\prime}$ and $17^{\prime}$ (mean $=9^{\prime}$ ) of arc for the three rightmost conditions. Individual results of 4 subjects, as well as their means and standard errors.

(bearing in mind its relatively small effects in Experiment 1) to determine whether it would have greater effects when the stimuli were not aligned with vestibular or retinal vertical. Both length conditions were run with gaps between the nearer line ends of $5^{\prime}, 10^{\prime}$, or $15^{\prime}$ of arc. In another condition, a single element length varying between $1^{\prime}$ and $7^{\prime}$ of arc (mean $\left.=4^{\prime}\right)$ and a gap of $1.5^{\prime}$ of arc were used. In addition, the possible effects of intrinsic orientation information were examined by making measurements at each gap size with two dots.

\section{Results}

The experimental results are shown in Figures 8 and 9. In Figure 8 are plotted all data from the two longer line lengths and vertical retinal orientation of the stimuli, with results for the different posture conditions grouped according to the gap between the line elements. Figure 9 shows data from the condition with a line length of $1^{\prime}-7^{\prime}$ of arc 

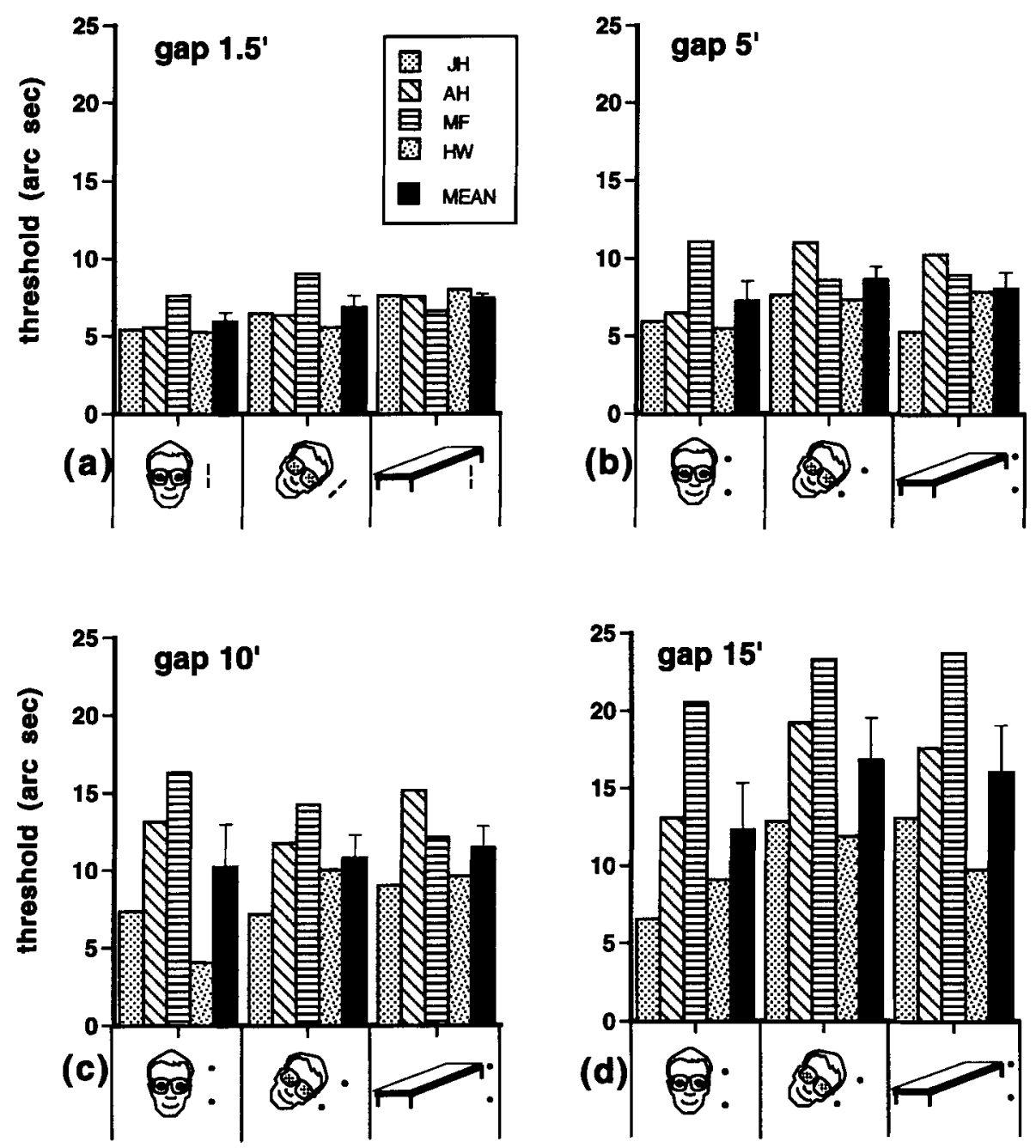

Figure 9. Results as in Figure 8, (i.e., vertical stimulus orientation) for line lengths between $1^{\prime}$ and $7^{\prime}$ (mean $=4^{\prime}$ ) of arc (Panel a) and for two-dot stimuli with three different gap sizes (Panels b, c, and d). Results from 4 individual subjects, as well as means and standard errors.

and a gap of $1.5^{\prime}$ of arc and for the different two-dot conditions. All conditions with retinally vertical stimulus orientation yielded very similar results for each stimulus type, with the best results for upright head position. The increase of thresholds for tilted head positions was less than around $20 \%$.

\section{Discussion}

The fact that all conditions with stimuli close to the retinal vertical orientation yielded very similar results indicates that this frame of reference is clearly most important. Variations of posture or of head orientation that remove the gravitational cue or usage of dot stimuli that do not contain an intrinsic reference have only a minor influence.

Experiment 4 was designed to further test this hypothesis of predominance of the retinal system of reference.

\section{EXPERIMENT 4 \\ Weak Retinal With (Mostly) Strong Gravitational and Varied Intrinsic Orientation Information}

Experiment 4 complemented the preceding one regarding the influence of the retinal versus the gravitational frame of reference. Experiment 3 supplied strong retinal with weak gravitational information, whereas Experiment 4 supplied weak retinal and (mostly) strong gravitational orientation information. Gravity, of course, was the same in both experiments, but, here, it could be used to discriminate between stimuli much better since the stimuli differed in the sign of their deviation from the vector of gravitation: clockwise versus counterclockwise. This type of discrimination is much more sensitive than the dis- 

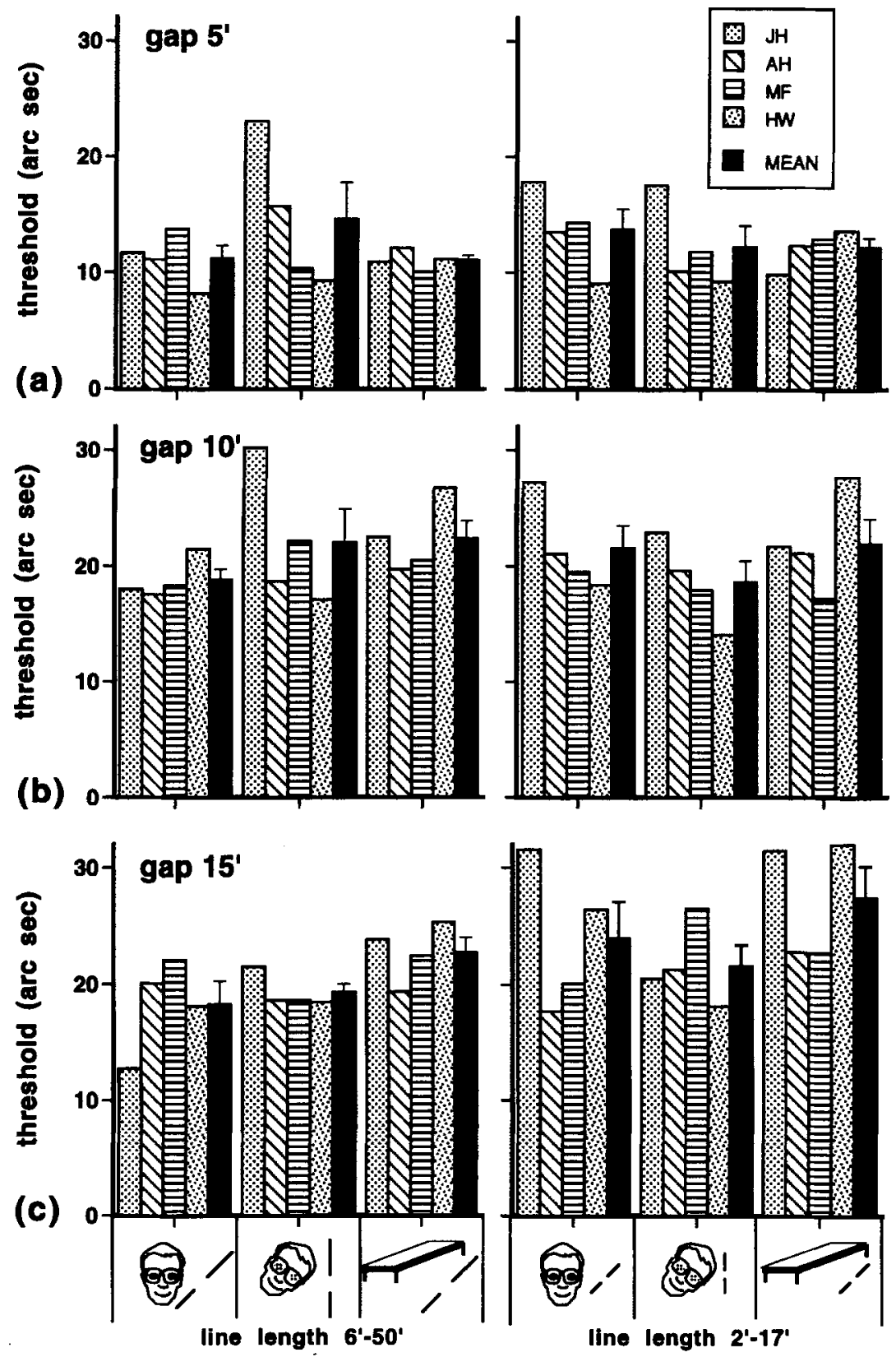

Figure 10. Results as in Figure 8, but stimuli were always obliquely oriented on the retina for long element lengths (left panels) and medium element lengths (right panels).

crimination between stimuli that are both oriented at about $45^{\circ}$ off the vector of gravitation, as was the case in Experiment 3 . The opposite is true for the retinal vertical: Stimuli in Experiment 4 were, on average, oriented at $45^{\circ}$ off the retinal vertical. Thus, the retinal system of reference supplied a much weaker cue than in Experiment 3: We are far less sure about when a line is oriented at $45^{\circ}$ than we are about whether or not it is oriented vertically.

\section{Method}

The apparatus, procedure, stimuli, and subjects were the same as those of Experiments 1-3. Both stimulus lengths of the line vernier stimuli were employed. The size of the vertical gap was $1.5^{\prime}, 5^{\prime}, 10^{\prime}$, or $15^{\prime}$ of arc. Moreover, two-dot stimuli were tested with interdot distances of $5^{\prime}, 10^{\prime}$, and $15^{\prime}$ of arc. We used the head-upright, headtilted, and supine conditions. Stimuli never coincided with retinal vertical, and they did or did not coincide with gravitational vertical.

\section{Results}

The experimental results are shown in Figures 10 and 11. Figure 10 summarizes the results for vernier line stimuli with the two longer element lengths. The left columns indicate results for the longer lines, and the right columns indicate results for the medium lines. Figure 11 shows the 

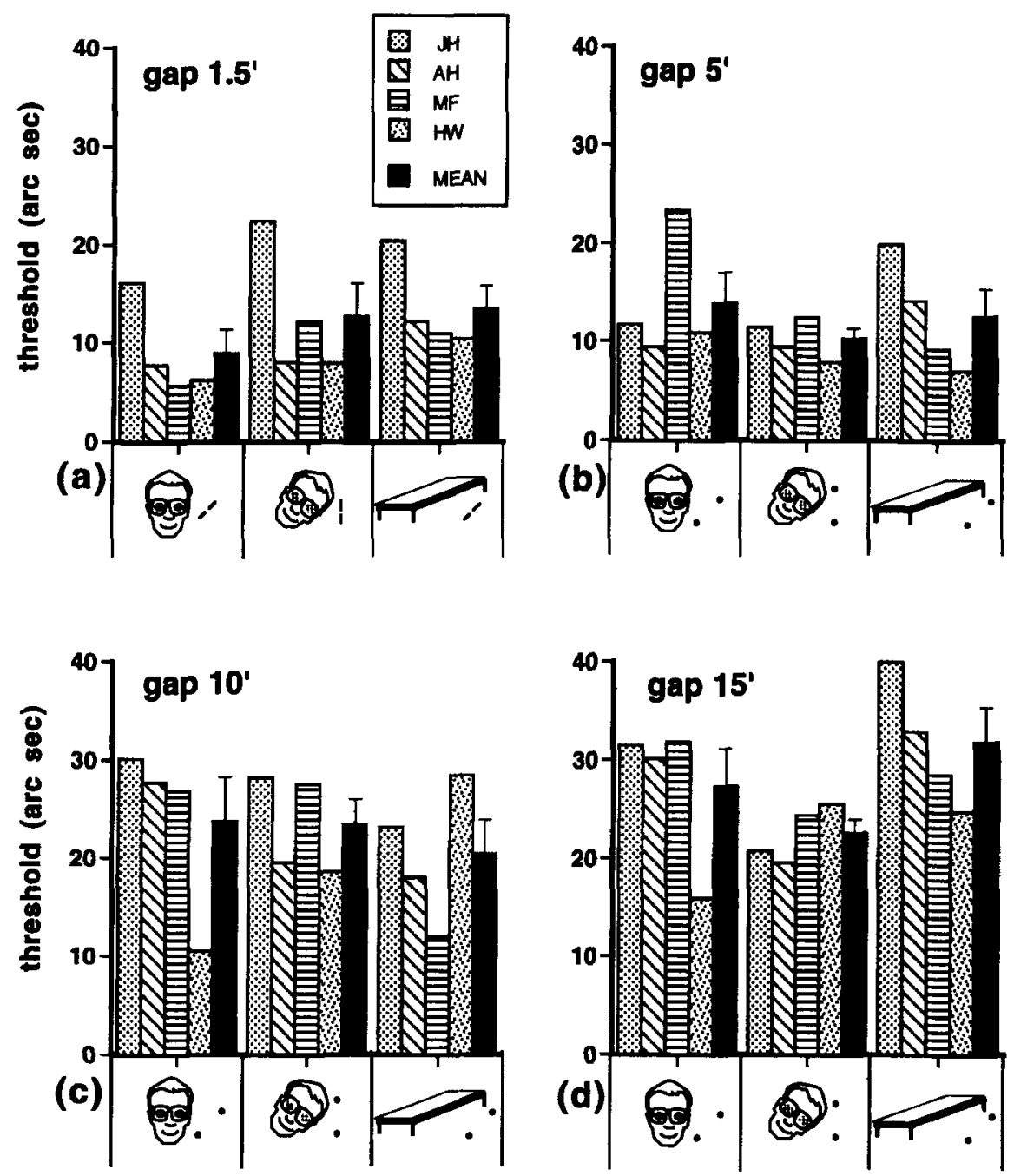

Figure 11. Results as in Figure 10, for line lengths between $1^{\prime}$ and $7^{\prime}\left(\right.$ mean $=4^{\prime}$ ) of arc (Panel a) and for two-dot stimuli with three different gap sizes. Results from 4 subjects, as well as means and standard errors.

results for the shortest line stimuli and for dot stimuli. All stimuli share the characteristic that they were oriented obliquely on the retina. Some of the stimuli coincided with gravitational vertical, whereas others did not. The results of all conditions were very similar, indicating that the presence of a strong gravitational cue does not have a major impact even in the absence of a strong retinal cue. Thresholds between the conditions again differed by about $20 \%$ or less.

\section{Discussion}

The results in Figures 8-11 demonstrate that the gravitational or subjective vertical does not exert an important influence: Thresholds for head and stimulus vertical were very similar to those for head and stimulus tilted by $45^{\circ}$ when the stimulus orientation on the retina was the same. This interpretation is supported by the data from the supine vernier vertical condition, for which the thresholds were almost identical to those from the head-upright con- dition. In the supine condition, the normal vestibular signal of the direction of gravitational vertical did not help to solve the visual task, but absence of this signal did not increase thresholds. This result strongly suggests that vestibular signals do not provide an important reference orientation in visual hyperacuity, and so the large mismatch between vestibular vertical and head position in the headtilted conditions should not affect those thresholds. The pattern of results from our head-upright and head-tilted conditions was very similar to that obtained by Corwin et al. (1977) from similar experimental manipulations. When their vernier target was vertical on the retina, thresholds were lower than when it was oblique, regardless of head position.

The important finding of Experiment 4 is that acuity was worse (by a factor of about 1.8 ) when the vernier was oblique on the retina than when it was retinally vertical, regardless of the position of the head, contrary to the results 


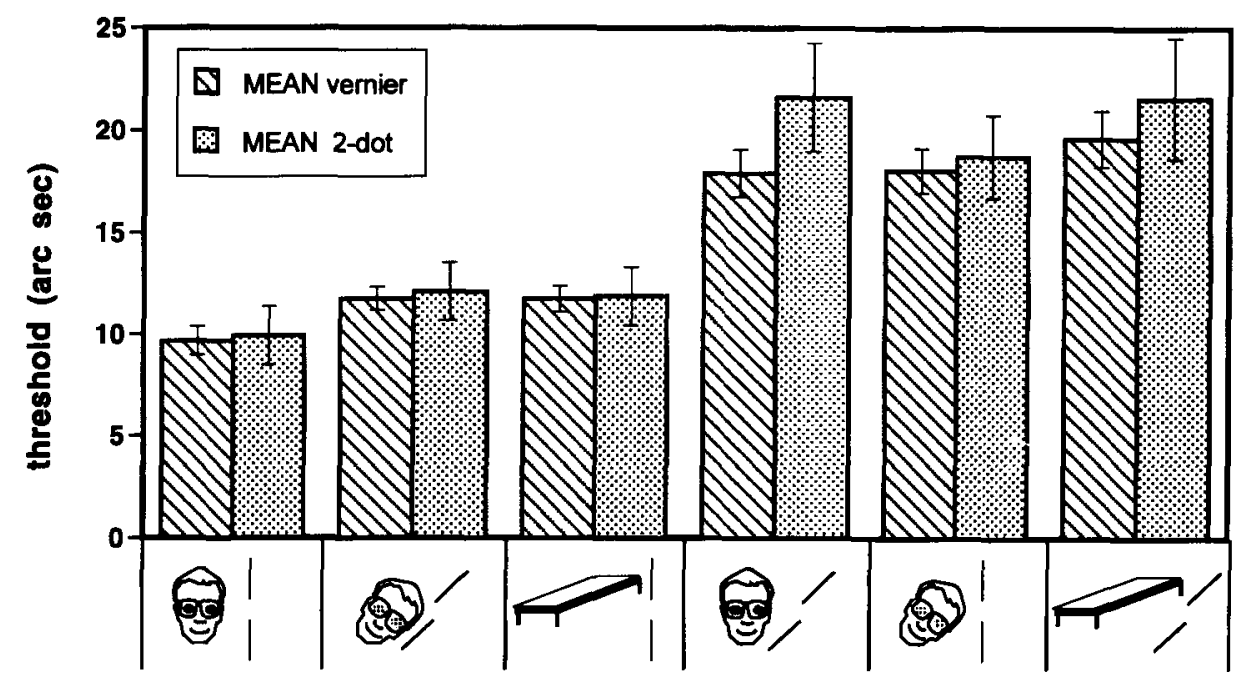

Figure 12. Results presented in Figures 7-11 collapsed over 4 subjects and two line and three gap sizes ("mean vernier"), and for two-dot stimuli ("mean dot"), collapsed over 4 subjects and three gap sizes. Vertical bars show standard errors.

reported for an orientation discrimination task (BuchananSmith \& Heeley, 1993). Thus, the relationship of the vernier to vestibular vertical is largely irrelevant, suggesting that this cannot be an important reference orientation for our task.

In Figure 12 are shown the mean thresholds for the six posture/retinal orientation conditions, obtained by collapsing the data from Experiments 3 and 4 over the two longer line lengths (maximum lengths of $50^{\prime}$ and $17^{\prime}$ of arc) and the three gaps $\left(5^{\prime}, 10^{\prime}\right.$, and $15^{\prime}$ of arc) and also separately for the two-dot conditions. It may be seen that the retinally vertical stimuli yielded thresholds of around $10^{\prime \prime}$ of arc, which were very similar to each other. Thresholds from the retinally oblique conditions were also similar to each other but higher (18" of arc or more) than those for the retinally vertical stimuli. For the retinally vertical stimuli, there was a slight tendency for thresholds to be larger when the head was not upright. Results for two-dot stimuli were very similar to those obtained with line stimuli.

\section{EXPERIMENT 5 \\ Possible Artifacts: Cyclotorsion and Frame Effects}

To check on the effects of possible errors in alignment of the head leading to incomplete compensation of the $45^{\circ}$ stimulus tilt by $\mathrm{a}^{\circ}$ head tilt due to possible cyclotorsion of the eyes (e.g., van Rijn, van der Steen, \& Collewijn, 1994), two control conditions were run with the head upright. Incomplete compensation would result in an orientation of the stimulus on the retina that would not correspond to the vertical meridian, but slightly off the vertical. In the first part of Experiment 5, the stimulus was tilted $10^{\circ}$ counterclockwise from vertical. In the second part, the stimulus was presented at an orientation randomly chosen from the range $10^{\circ}$ clockwise to $10^{\circ}$ counterclockwise from vertical. The stimuli in these conditions had one of the range of line lengths used above, and a gap of $1.5^{\prime}$ or $15^{\prime}$ of arc. In a final control, we addressed the possible role of the frame of the monitor as an additional system of reference by adding a circular aperture in front of the monitor that masked the monitor's frame.

\section{Method}

The experimental setup and procedure were the same as in Experiments 1-4. Vernier line stimuli were presented either at a constant orientation $10^{\circ}$ clockwise or counterclockwise from the vertical or at orientations randomly chosen from the range between $10^{\circ}$ clockwise to $10^{\circ}$ counterclockwise from vertical. In the third part of the experiment that tested the possible role of the system of reference supplied by the borders of the monitors, the subjects looked at the monitor through a tube (with a diameter of $10 \mathrm{~cm}$ and a length of $2 \mathrm{~m}$ ) that completely obscured the borders of the monitor. The part of the monitor outside of the tube was masked by means of a circular aperture.

\section{Results}

Figure 13 shows the effect, with the head upright, of presenting the vernier either at a constant tilted orientation of $10^{\circ}$ from vertical or at variable orientations. These thresholds should be compared with the left-hand bar in Figure 12, in which both the head and the vernier target were upright. The effect of tilting the vernier by a constant amount was to increase thresholds by a factor of 1.7 for short stimuli (Figure 13a), and the effect of presenting it at a randomly chosen angle was to increase thresholds by a factor of 2.3 for short stimuli (Figure 13a) and by a factor of 1.1 for long stimuli (Figure 13b). The effects of the constant orientation offset were negligible for the long stimuli. In fact, thresholds were even slightly reduced in this condition. The result indicates that the higher susceptibility of shorter verniers to variable stimulus orientation also holds true for our group of subjects. 

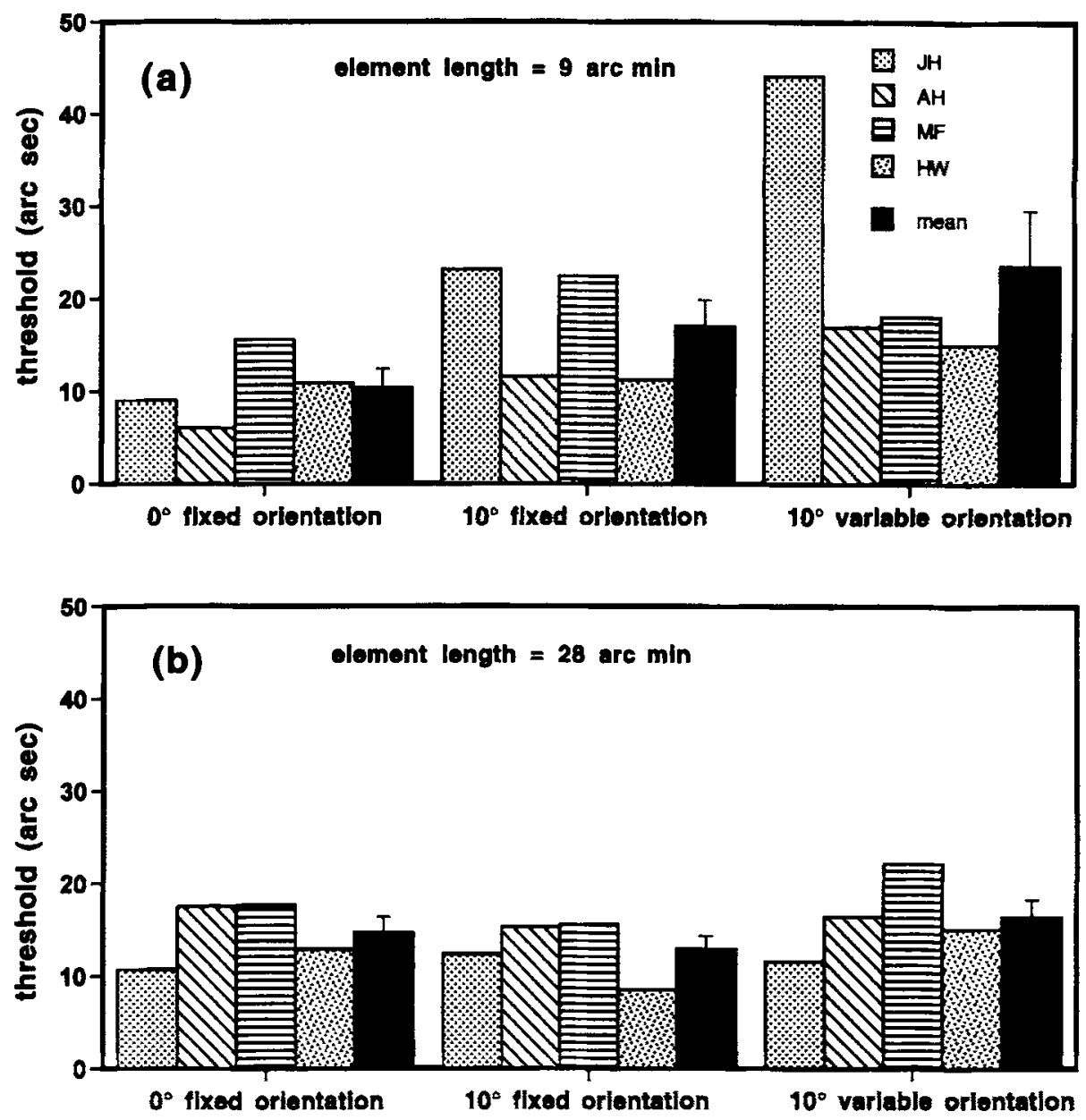

Figure 13. Control experiment to evaluate the possible role of an incomplete compensation of stimulus tilt by head tilt. Vertical gap size was $15^{\prime}$ of arc. Stimulus orientation was constant at $0^{\circ}$, constant at $10^{\circ}$, or varied randomly by $\pm 10^{\circ}$ around the vertical. Vertical bars show standard errors.

The purpose of a final control experiment was to exclude the possible effects of the monitor's borders serving as a reference frame. This experiment was essentially a replication of a part of Experiment 2, in which we measured the oblique effect both with collinear verniers and with two dots. For this control, a circular aperture of $3^{\circ} \mathrm{di}-$ ameter masked the periphery of the monitor, and the subjects gazed at the monitor through a long pipe. The results were not significantly affected by these measures (see Figures 14 and 15) - that is, the oblique effect was at least as pronounced in the absence as it was in the presence of the monitor frame (see Figures 6 and 7).

\section{Discussion}

We found that retinal vertical is the most (if not the only) important orientation parameter in vernier discriminations. Other orientation cues, such as subjective or gravitational vertical, that may be considered as "higher level" influences on orientation perception do not play an important role. The slight advantage of keeping the head up- right, compared with the other posture conditions as found in some instances, may reflect the fact that, in the latter position, the orientation of the head no longer coincides with gravitational vertical, which could provide a useful reference orientation. However, another possibility is that the head and, therefore, the retina are not perfectly aligned with the vernier in the conditions in which the head is not upright. The exact alignment between stimulus and retinal vertical may be difficult to achieve because, even if the head is perfectly aligned, cyclotorsion of the eyes may produce a mismatch between retina and stimulus, though this mismatch was probably small under our experimental conditions without rich visual stimulation.

When the orientation of the short vernier had a small systematic deviation from vertical, or its orientation was jittered around vertical (Figure 13), thresholds rose slightly, relative to the case in which both head and vernier were vertical. For longer stimuli, on the other hand, small deviations of stimulus orientation did not show a significant influence on thresholds. The system of reference provided 

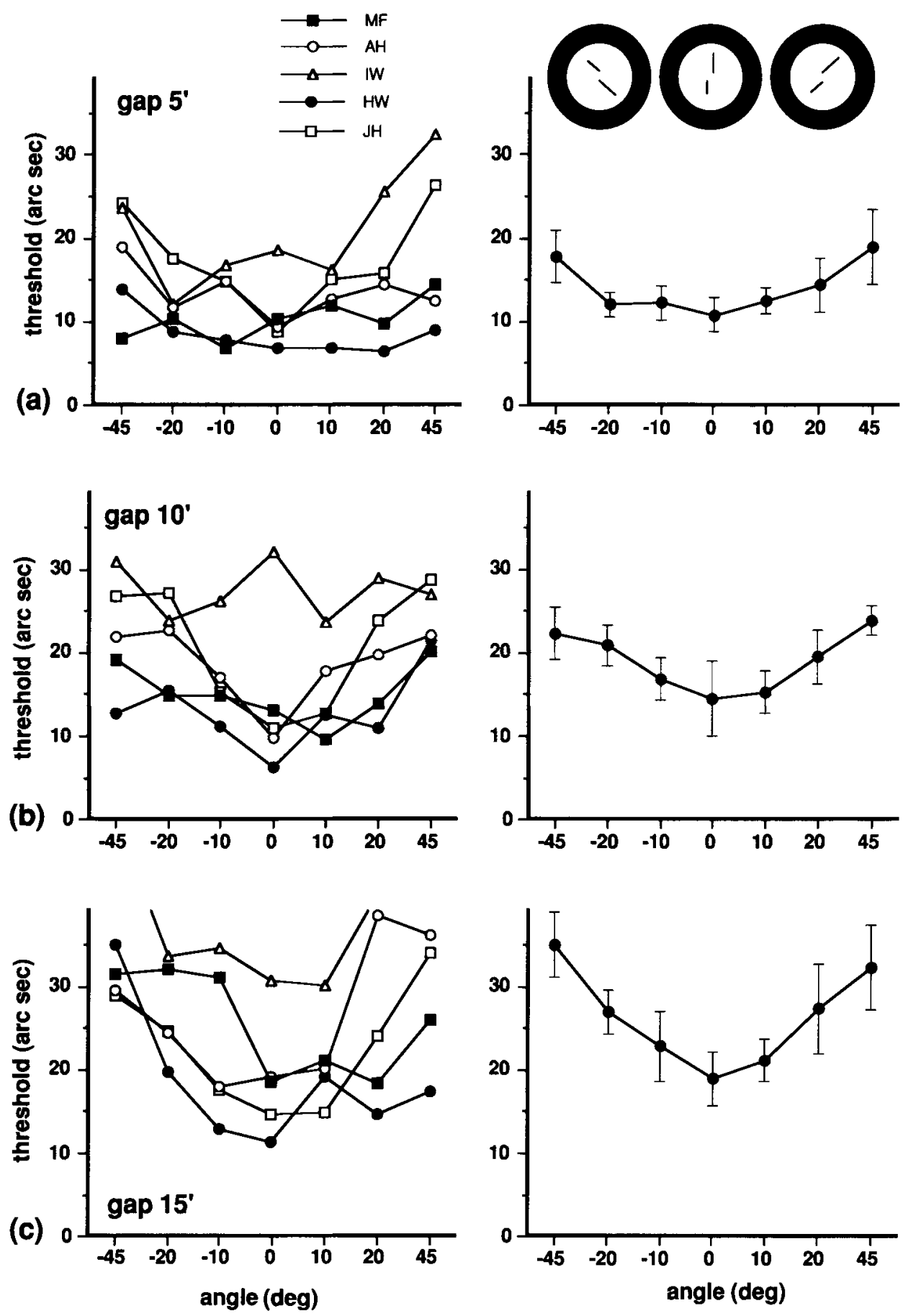

Figure 14. Vernier thresholds for collinear line targets as a function of stimulus orientation. The individual results of 5 observers are shown in the left-hand panels; their means and standard errors are shown in the right-hand panels. The stimuli were viewed with the head upright through a circular tube that prevented the perception of any vertical or horizontal edges on the monitor or in the testing room. Note the similarity of the results to those presented in Figure 6.

by the frame of the monitor did not play an important role: Masking this frame did not influence thresholds in our experiments and in those by Saarinen and Levi (1995), who used a tilted rectangular rather than a circular frame of reference in their control condition.

\section{GENERAL DISCUSSION}

\section{Summary of the Results}

The starting point for this study was the idea that a vernier acuity task can involve orientation judgments, as is often 

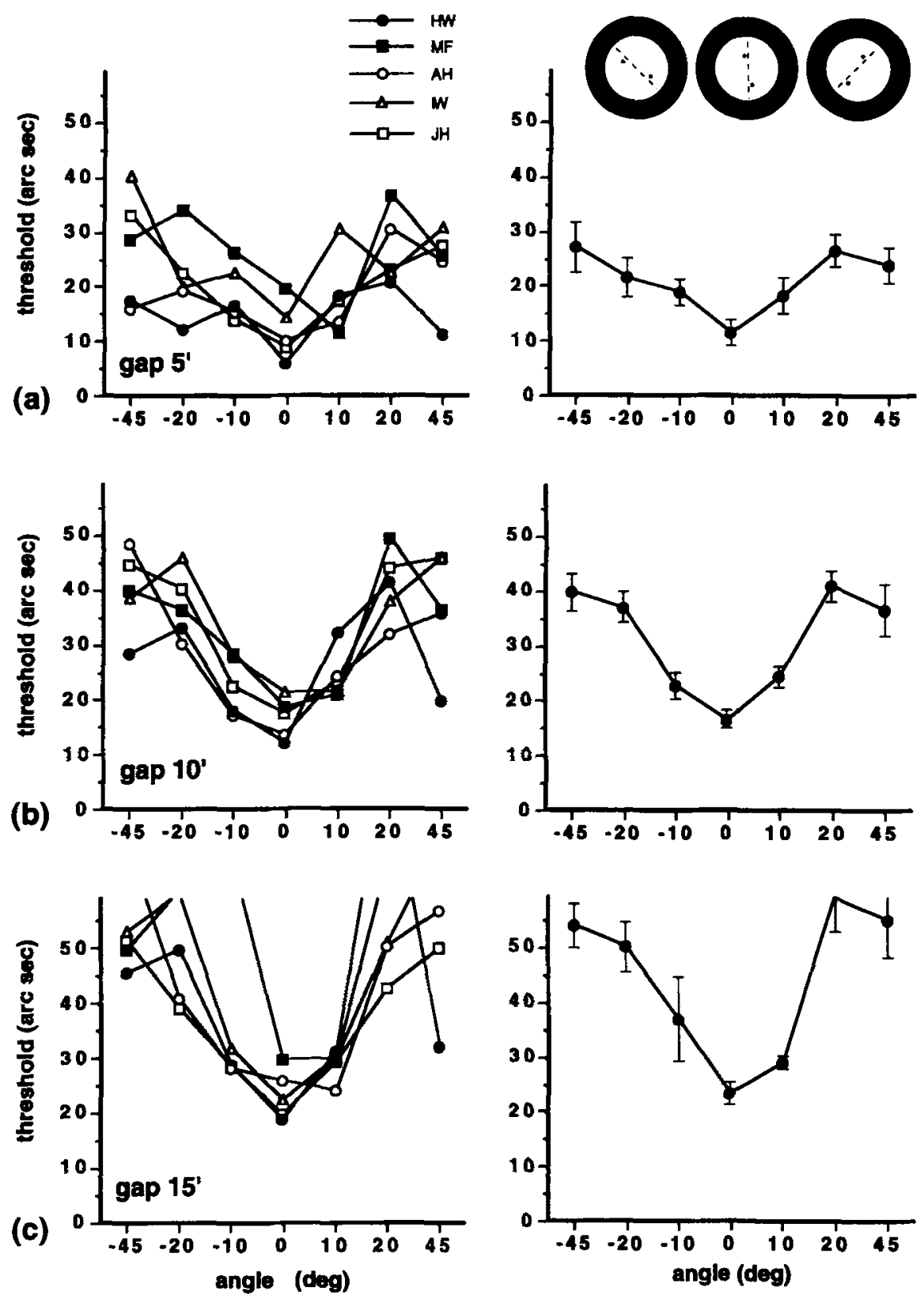

Figure 15. Vernier thresholds for two-dot targets as a function of stimulus orientation. The individual results of 5 observers are shown in the left-hand panels; their means and standard errors are shown in the right-hand panels. The stimuli were viewed with the head upright through a circular tube that prevented the perception of any vertical or horizontal edges on the monitor or in the testing room. Note the similarity of the results to those presented in Figure 7.

suggested (Sullivan et al., 1972; see Morgan, 1991, for review). Our question concerned the nature of the possible reference orientations to which an implicit line through a vernier stimulus or an implicit line joining its line ends might be compared and whether the dependence of orientation judgments on subjective vertical (Buchanan-Smith \& Heeley, 1993) would hold true for vernier discriminations. In this study, we evaluated the relative importance of three reference orientations: retinal vertical, vestibular vertical, and stimulus-defined, or intrinsic, vertical.

The data from the present study may be summarized as follows. When the line elements of a vertical vernier are rotated around their near endpoints in chevron fashion, thresholds rise as the deviation of the lines from straightness increases. This is true whether the offset is horizontal and rotation of the lines from vertical makes them oblique 
or whether the offset is at $45^{\circ}$ from vertical and rotation of the lines makes them horizontal and vertical. These effects of rotating the vernier segments, so that the angle between them and the direction of the offset change, are larger for longer lines, suggesting that they provide more salient misleading orientation information. When the vernier elements are reduced to two dots at their near ends, thresholds are comparable to those found for parallel line elements at vertical orientation, although they are slightly but significantly higher at oblique orientations. With oblique presentations, thresholds rise as a vernier with parallel line elements increasingly deviates from vertical.

The subject's posture is relatively unimportant in vernier acuity: Thresholds measured with the head vertical, with the head tilted at $45^{\circ}$ to vertical, and with the subject lying supine are very similar, provided the vernier target is vertical on the retina. Thresholds rise by about the same amount for all three postures if the vernier is presented obliquely on the retina. The increase of thresholds with deviation from vertical is comparable for short and long stimuli and for stimuli with different vertical gap sizes.

\section{Possible Nonretinal Reference Orientations}

Two possible nonretinal candidate reference orientations in the typical vernier acuity study - in which the vernier stimulus is straight, the segments are almost collinear, and the subject's head is upright-are stimulus-defined and vestibular vertical. The oblique effect on vernier acuity (confirmed in Experiment 2) or the rise in thresholds in our chevron-like figure (Experiments 1 and 2) might occur either because the stimulus increasingly deviates from retinal and vestibular vertical, rendering a comparison with these main cardinal axes more difficult, or because stimulus orientation is less well defined-that is, detection of orientation is more "noisy" for these orientations (the oblique effect). However, the condition in Experiment 2 in which the quasi-chevron was rotated by $45^{\circ}$ produced the highest thresholds when its target lines were vertical and horizontal. This result indicates that the increase of thresholds found in Experiment 1 is not due simply to the oblique effect (e.g., less precise detection of orientation at oblique angles) but is caused by the fact that the orientations of the segments do not coincide.

In Experiment 3, thresholds rose by only a small amount when both the head and the vernier were tilted, and this marginal effect could have been produced by a slight misalignment of retina and vernier. Taken together, these results suggest only a minor role, if any, for gravitational vertical in vernier acuity, in agreement with the results of Saarinen and Levi (1995). Similarly, the results from the two-dot stimuli, relative to the line stimuli (see, e.g., Figures 3, 4, $5,7,12$, and 15), suggest that the intrinsic reference potentially provided by vernier targets does not normally make any significant contribution to vernier acuity of sufficiently long stimuli if orientation is vertical (i.e., in the presence of the retinal vertical reference). However, rotation of the separate lines of the vernier target into a quasi- chevron can provide potentially misleading or interfering intrinsic reference orientations.

\section{Origin of the Oblique Effect}

The important determinant of thresholds in all experiments was the relationship between the retina and the orientation of the stimulus. The results of Experiment 1 on the quasi-chevrons (Figures 3 and 4) and of Experiment 2 on the conventional oblique effect (Figures 6 and 7) could be interpreted solely in terms of the properties of the lines in the target. As these became more oblique on the retina, thresholds rose. This finding would be in line with a large body of results on the oblique effect, including hyperacuity (for a recent review, see Saarinen \& Levi, 1995, who also show that the oblique effect in hyperacuity is not caused by lower contrast sensitivity for oblique orientations).

The data from Experiment 2 (Figure 5) on the rotated quasi-chevron show that this cannot be a complete explanation as mentioned above. In Experiment 2, the highest thresholds were associated with vertical and horizontal target lines, and the lowest with oblique lines (i.e., contrary to the predictions of the oblique effect). What also matters is how the direction of offset is given. If this is horizontal (and so at $90^{\circ}$ to retinal vertical; Figures 3 and 4 ) or is at $90^{\circ}$ to the target lines (whether or not these are vertical; Figures 6 and 7), then performance seems to be governed by whether lines are oblique on the retina. However, when the offset is oblique (Figure 5) and so at $45^{\circ}$ to retinal vertical, performance seems to be governed by its relationship to the target lines. If this is $90^{\circ}$ (cf. Figure $5 ; 0^{\circ}$ ), so specifying direction most precisely, thresholds are lower than when it is at $45^{\circ}$ (Figure $5 ; 45^{\circ}$ ), even though the target lines are retinally oblique in the former case. Thus, there seem to have been two separable oblique effects operating in these experiments, one of line orientation and one of offset direction or of angle between the vernier's segments.

It appears that both effects could be accommodated within a line element model (Wilson, 1986), provided that obliquely oriented receptive fields had a less precise orientation tuning than vertical or horizontal receptive fields and that our chevron-like stimuli were processed by receptive fields whose long axis was orthogonal to the vernier offset (see Figures $2 \mathrm{c}$ and $2 \mathrm{~d}$ ). Thresholds for the chevronlike stimuli were always lower, at identical intersegment angles, for vertical overall orientation (Figures 3 and 4) than for oblique overall orientation (Figure 5; note change of scale in ordinate). Thus, thresholds would be higher for oblique than for horizontal offsets, but superimposed on this fall in acuity can be an additional component produced by tilting the target lines into a quasi-chevron and so positioning them in the inhibitory flanks of the receptive fields.

\section{Memory Effects: Building an Internal Standard}

The chevron-like figure of Experiment 1 and the conventional vernier of Experiment 2 yielded very similar patterns of results as the line elements became more oblique. However, in Experiment 2, an oblique effect similar to that 
with lines was found when the lines were reduced to dots at their near endpoints. As noted in the Discussion section of Experiment 2, a single presentation of a pair of dots is ambiguous. The subject cannot know whether the dots represent a large deviation from one orientation or a small deviation from another. This ambiguity can be solved only by comparing the appearance of successive stimuli and constructing (at least) one internal template or standard with which to compare the external stimuli. Since the direction of the offset is explicit in a line vernier but only implicit in a dot vernier, the similarity of the results from oblique line and dot verniers is perhaps surprising. However, a similar effect of implicit target attributes has been reported by Westheimer and McKee (1977) and Morgan (1992). It is reassuring, though, that loss of the intrinsic reference when replacing the oblique line verniers by dots leads to a significant, if small, increase of thresholds.

\section{Possible Monitor Frame Effects}

One possible visual frame of reference in all our experiments was the rectangular frame of the monitor itself. It could be argued that this contributed to the various effects reported here. For example, the change in thresholds as the angle varied between the lines of the quasi-chevron (Experiment 1) could have arisen not because of the changes in retinal orientation but because of the systematic variation in the orientation between the target lines and the edges of the monitor. However, several lines of evidence suggest that such monitor frame effects were very small, if they existed at all, in our conditions.

First, the stimuli in Experiment 1 were obtained by holding frame orientation constant and by electronically varying the rotation of the target lines, whereas the orientation of the collinear stimuli in Experiment 2 was varied by keeping stimulus orientation on the monitor constant and by varying monitor orientation. Despite the very different relationships between target lines and frame edges in the two conditions, very similar effects of changing retinal orientation were found. These results agree well with those obtained by Saarinen and Levi (1995), indicating that there are no significant higher level influences on orientation anisotropy in vernier acuity tasks, as opposed to orientation discrimination tasks (Buchanan-Smith \& Heeley, 1993).

Second, in Experiment 3, stimulus orientation was always varied by rotating the monitor. Thus, the target lines were always parallel to the sides of the monitor. Despite this unvarying frame information, clear effects of retinal orientation were found.

Finally, we have found that the effects of varying the retinal orientation of a collinear target with the head upright remain similar to those reported in Figures 6 and 7 when the edges of the monitor frame, and other possible visual cues to vertical and horizontal, cannot be used. In these control experiments, the stimuli were presented in the middle of a circular aperture and viewed through an internally blackened circular tube that obscured any vertical or horizontal edges in the room. These data are shown in
Figures 14 and 15 , and are very similar to those presented in Figures 6 and 7 . Thus, we conclude that our data do not reflect the relationship between the orientation of the target lines and that of other lines or edges.

\section{Conclusions}

In principle, various sources of orientation information might influence the precision of vernier acuity. From experiments in which the orientation of the subject and of the verniers was altered, we conclude that vestibularly signaled gravitational vertical is not an important reference for this type of visual task. The intrinsic orientation information provided by the typical vernier stimulus seems to be slightly more important, as is obvious from the difference in results between obliquely oriented dots and line stimuli. In contrast, vernier performance is progressively impaired as the stimulus deviates more from retinal vertical, provided that the direction of the offset to be discriminated is well defined. When the offset is oblique, and not at $90^{\circ}$ to the target lines, thresholds can be higher when the target lines are horizontal and vertical on the retina than when they are oblique. Hence, the most important reference for vernier discrimination is retinal vertical, with performance strongly deteriorating with increasing angle of the stimulus from vertical (or horizontal). Thresholds increase even more strongly if the segments of the vernier are not parallel but differ in orientation, in good agreement with a model that line element detectors with antagonistically organized center-surround organization might underlie offset discrimination in vernier stimuli.

\section{REFERENCES}

Andrews, D. P., Butcher, A. K., \& Buckley, B. R. (1973). Acuities for spatial arrangement in line figures: Human and ideal observers compared. Vision Research, 13, 599-620.

BanKS, M. S., \& Stolarz, S. J. (1975). The effect of head tilt on meridional differences in acuity: Implications for orientation constancy. Perception \& Psychophysics, 17, 17-22.

Buchanan-Smith, H. M., \& Heeley, D. W. (1993). Anisotropic axes in orientation perception are not retinotopically mapped. Perception, 22, 1389-1402.

Corwin, T. R., Moskowitz-Cook, A., \& Green, M. A. (1977). The oblique effect in a vernier acuity situation. Perception \& Psychophysics, 21, 445-449

FinNey, D. J. (1962). Probit analysis. Cambridge: Cambridge University Press.

HARRIS, J. P., \& FAHLE, M. (1995). The detection and discrimination of spatial offsets. Vision Research, 35, 51-58.

LENNIE, P. (1974). Head orientation and meridional variations in acuity. Vision Research, 14, 107-111.

Morgan, M. (1991). Hyperacuity. In D. Regan (Ed.), Spatial vision: Vol. 10. Vision and visual dysfunction (pp. 87-113). London: Macmillan.

Morgan, M. (1992). On the scaling of size judgements by orientational cues. Vision Research, 32, 1433-1445.

Orban, G. A., Vandenbussche, E., \& Vogels, R. (1984). Human orientation discrimination tested with long stimuli. Vision Research, 24, $121-128$.

REgan, D., \& Price, P. (1986). Periodicity in orientation discrimination and the unconfounding of visual information. Vision Research, 26, 1299-1302.

SAARINEN, J., \& LEVI, D. M. (1995). Orientation anisotropy in vernier acuity. Vision Research, 35, 2449-2461. 
Sullivan, G. D., OAtley, K., \& Sutherland, N. S. (1972). Vernier acuity as affected by target length and separation. Perception \& Psychophysics, 12, 438-444.

TAYlor, M. M., \& CReelman, C. D. (1967). PEST: Efficient estimates on probability functions. Journal of the Acoustical Society of America, 41, 782-787.

van Rijn, L. J., van der Steen, J., \& Collewijn, H. (1994). Instability of ocular torsion during fixation: Cyclovergence is more stable than cycloversion. Vision Research, 34, 1077-1087.

WATT, R. J. (1984). Towards a general theory of the visual acuities for shape and spatial arrangement. Vision Research, 24, 1377-1386.

WATT, R. J., \& CAMPBELL, F. W. (1985). Vernier acuity: Interactions between length effects and gaps when orientation cues are eliminated. Spatial Vision, 1, 31-38.
WatT, R. J., MORGaN, M. J., \& WARD, R. M. (1983). The use of different cues in vernier acuity. Vision Research, 23, 991-995.

WESTHEIMER, G. (1985). The oscilloscopic view: Retinal illuminance and contrast of point and line targets. Vision Research, 25, 1097-1103.

WESTHEIMER, G., \& MCKEE, S. P. (1977). Spatial configurations for visual hyperacuity. Vision Research, 17, 941-947.

WILSON. H. (1986). Responses of spatial mechanisms can explain hyperacuity. Vision Research, 26, 453-469.

(Manuscript received March 7, 1996; revision accepted for publication February $25,1997$. 